Article

\title{
Benchmarking of Dedicated Hybrid Transmissions
}

\author{
Christian Sieg * and Ferit Küçükay \\ Institute of Automotive Engineering, Technische Universität Braunschweig, 38106 Brunswick, Germany; \\ f.kuecuekay@tu-braunschweig.de \\ * Correspondence: c.sieg@tu-braunschweig.de; Tel.: +49-531-391-2641
}

Received: 20 December 2019; Accepted: 10 February 2020; Published: 13 February 2020

\begin{abstract}
For many manufacturers, hybridization represents an attractive solution for reducing the energy consumption of their vehicles. However, electrification offers a wide range of possibilities for implementing powertrain concepts. The concepts can differ regarding their mechanical complexity and the required power of the electrical machines. In this article, drive concepts that differ in their functionality and drive train topology are compared. Based on requirements for the $\mathrm{C}, \mathrm{D}$, and $\mathrm{E}$ segment, the mechanical and electrical effort of the concepts is analyzed. The results show that the mechanical effort in the $\mathrm{C}$ segment can be reduced as long as the electrical effort is increased. In case of higher vehicle segments, the electrical effort can increase considerably, making concepts with increased mechanical complexity more suitable. The driving performance and efficiency in hybrid operation are evaluated via simulation. The results show that the difference of acceleration times in hybrid operation between a charged and discharged battery is lower for mechanically complex concepts. At the same time, they achieve lower $\mathrm{CO}_{2}$ emissions. Therefore, these concepts represent a better compromise regarding performance and efficiency. Despite lower transmission efficiencies in hybrid operation, they achieve conversion qualities similar to simpler concepts and lower emissions with lower electrical effort.
\end{abstract}

Keywords: dedicated hybrid transmission; benchmarking; hybrid electric vehicle; efficiency; topology optimization; drive train optimization; powertrain concepts

\section{Introduction}

To reduce the energy consumption, electrification of the drive train represents a suitable solution. Since one or more electric machines (EM) can be integrated into the drive train at different positions, a large number of possible drive concepts for hybrid electric vehicles (HEV) can be realized. The possible drive trains differ in their characteristics and operating modes. On the one hand, powertrains of conventional vehicles can be electrified, resulting in comparatively complex mechanical concepts. On the other hand, it is possible to implement transmissions that are dedicated for use in HEV and have a much simpler design.

Due to this diversity, the question which kinds of drive train concepts represent suitable solutions in which vehicle segment needs to be answered. It is of interest to be able to make a statement whether the mechanical effort of a transmission can be reduced in low vehicle segments without having to accept a loss of driving performance or efficiency. At the same time, the question should be answered whether higher vehicle segments require an increase in the mechanical complexity of the transmission. The aim of this article is to answer these questions. A detailed analysis regarding the influence of multi-speed transmissions on various basic dedicated hybrid transmission (DHT) concepts is carried out by [1]. However, coaxial multi-mode DHT (MM-DHT) with several planetary gear sets (PGS), which enable parallel as well as power-split operating modes, are not included in the considerations.

For these reasons, this article compares drive train concepts with different mechanical and electrical complexity. Several add-on concepts as well as DHT are considered. Furthermore, similar concepts 
with different mechanical complexity are analyzed. Based on equal driving performance requirements for all concepts, it is shown which EM power is necessary to meet the requirements for plug-in hybrid electric vehicles (PHEV) in the $\mathrm{C}, \mathrm{D}$, and E segments. The results confirm the correlation between the concept's mechanical and electrical complexity identified by [1]. They show that a reduction of the mechanical complexity is only possible in connection with a simultaneous increase of the electrical power. Based on the results, it can be derived which concepts can be used in which segment. Furthermore, characteristic properties of the considered concepts are derived.

Within the scope of a concept comparison for vehicles in the $C$ segment, the concepts are evaluated regarding their efficiency in hybrid operation and driving performance. Apart from these factors, especially costs for mechanical and electrical components as well as the necessary installation space have a major influence on the concept decision. However, the costs depend on the quantities of produced transmissions. In addition, they may be influenced by using existing components from either previous or other vehicle models so that the costs of a powertrain concept are manufacturer-specific. Furthermore, not every component is produced by vehicle manufacturers so that the transmission costs also depend on the suppliers. To assess the required installation space, a detailed design of the transmission is necessary. Therefore, the mechanical components such as gears, bearings, shafts, and the transmission housing need to be dimensioned regarding worst-case scenarios, which are unknown for new concepts in early development phases and can only be estimated. In addition, the available installation space depends on a specific vehicle model. In early concept phases, it is unknown in which segment or vehicle a powertrain concept will be used. Considering the legislation, it can be expected that the energy consumption of vehicles needs to be reduced even further in the future. Thus, it is a major influencing factor when comparing powertrain concepts. For these reasons, this paper focuses on efficiency and driving performance.

The results show that concepts with high mechanical effort offer a good compromise between driving performance and efficiency. The advantage of these concepts is that in hybrid operation there are minor differences in acceleration times when the battery can be discharged or needs to be charged. If the concepts are simpler, a higher share of the power of the internal combustion engine (ICE) must be transmitted via the EM, resulting in higher losses and thus lower driving performance.

\section{Drive Train Concepts}

In this article, different powertrain concepts for hybrid vehicles are compared. The topologies differ considerably regarding their structure, the number of EM, the power flows occurring in the drive train and the operating modes. The mechanical and electrical complexity of the concepts is another important distinguishing feature.

Hybrid powertrains can be divided into two main categories. In addition to so-called add-on hybrids based on conventional powertrains, this article examines DHT especially designed for use in hybrid vehicles. Furthermore, hybrid drive trains can be divided into parallel hybrids, series-parallel hybrids, power-split hybrids, and multi-mode DHT (MM-DHT).

\subsection{Parallel Hybrid Concepts}

Due to their high market share, two parallel hybrids are analyzed in this article. They are based on a conventional drive train and differ with respect to the positioning and the number of EM. In addition to the P2 topology, explained in Section 2.1.1, a P1P4 topology, shown in Section 2.1.2, with two EM is investigated. In both concepts, an 8-speed transmission with a wet dual-clutch is used.

\subsubsection{P2 HEV}

The drive train topology of a P2 hybrid is shown in Figure 1. In this configuration, an EM is positioned between the ICE and the transmission. Furthermore, there is a separating clutch between EM and ICE, so that the ICE can be disconnected from the drive train. Thus, the vehicle can be driven either electrically without ICE drag losses or by the combustion engine solely or in hybrid mode. 


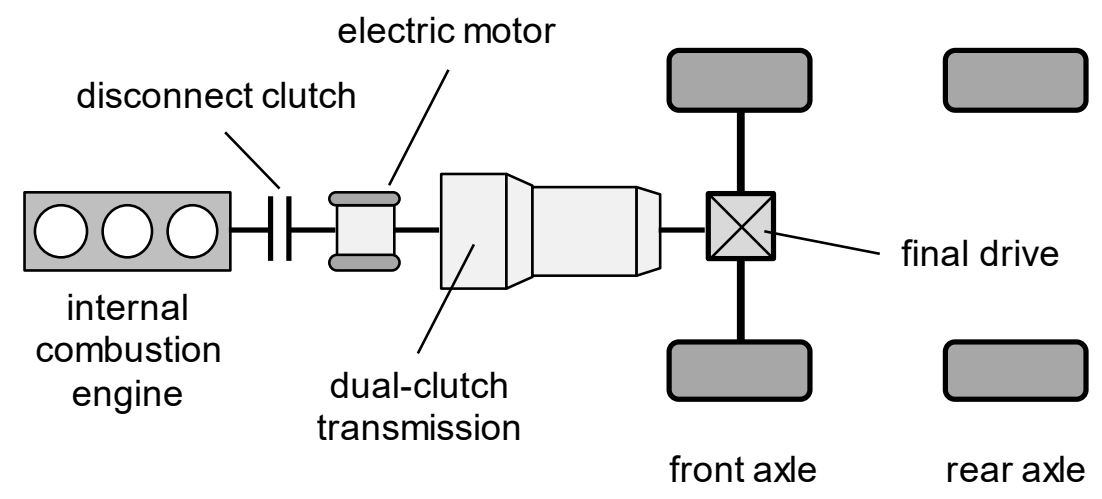

Figure 1. P2 drive train topology.

The transmission of this add-on topology is a wet 8-speed dual-clutch transmission (DCT). Consequently, it offers eight hybrid and eight electric driving modes.

Since P2 HEV are based on conventional drive trains, they are characterized by a high number of mechanical components compared to other concepts considered in this paper. To estimate and compare the mechanical effort of different concepts, the number of relevant mechanical components can be totaled. In the P2 HEV from Figure 1, the relevant components include the double clutch at the transmission input, the separating clutch between ICE and EM, the final drive (FD) at the front axle and the eight gears. This results in a total mechanical effort of 11 .

\subsubsection{P1P4 HEV}

Apart from P2 HEV, vehicles with an electrified rear axle, so-called P4 hybrids, show a high market share. Thus, a parallel hybrid with an electrified rear axle is investigated in this paper, see Figure 2. To enable battery charge at standstill and to start the ICE quickly and comfortably while driving, the drive train additionally comprises an electric motor between ICE and transmission. Since there is no disconnect clutch, electric driving is only possible by using the rear axle.

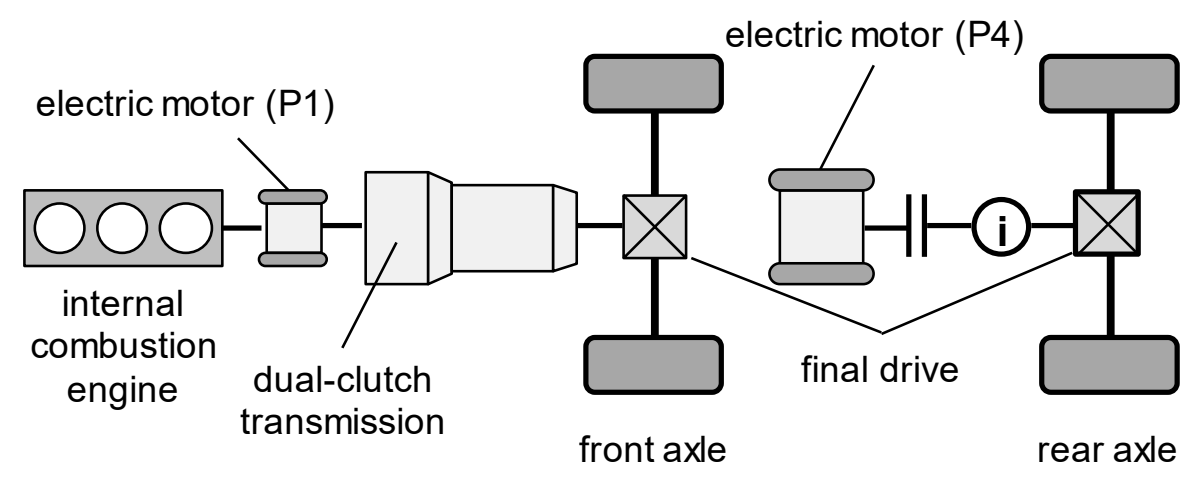

Figure 2. P1P4 drive train topology.

Because of the positioning of the electric motor on the rear axle, the P1P4 topology offers temporary all-wheel drive. To avoid over-speeding of the rear electric motor, a disconnect device is installed between the EM and the reduction gear on the rear axle. The concept comprises the same 8-speed DCT as the P2 HEV concept. It offers an electric driving mode and 16 hybrid modes since the vehicle can be driven in eight gears with or without the rear axle.

Similar to the P2 hybrid, the P1P4 hybrid has a high mechanical complexity. Both the eight gears of the transmission and the FD on the front axle are identical to the P2 concept. Differences result from the fact that there is no separating clutch between the ICE and the P1 EM and that the EM is connected to a second FD at the rear axle via a reduction gear with a disconnect device. This results in a higher mechanical effort of 13 compared to the P2 HEV. 


\subsection{Series-Parallel DHT}

Another topology suitable for HEV is the powertrain shown in Figure 3. On the one hand, it enables a serial operating mode in which there is no mechanical connection between the ICE and the wheel, and the EM can transmit the power electrically. On the other hand, the ICE and the wheel can be connected by closing a separating clutch so that the ICE drives the vehicle at higher wheel powers. When the clutch is open, an electrical mode is possible. The shift matrix of the concept is shown in Table 1.

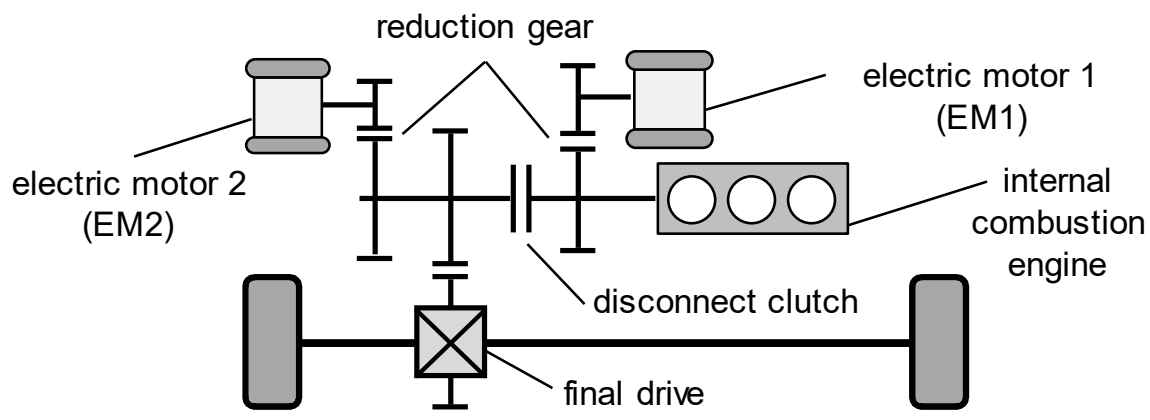

Figure 3. Drive train topology of the series-parallel DHT.

Table 1. Shift matrix of series-parallel DHT.

\begin{tabular}{cc}
\hline Operating Mode & C \\
\hline Electric & \\
Parallel & $\bullet$ \\
Serial hybrid & \\
\hline
\end{tabular}

The series-parallel concept differs greatly from the other concepts in this article in terms of its design, operating modes, and mechanical complexity.

The mechanically relevant components include the two reduction gears, the separating clutch and the FD, so that the mechanical effort of 4 is lower compared with most other concepts.

\subsection{Power-Split DHT Concepts}

In addition to add-on hybrids, the Toyota Prius' power-split hybrid drive system has established itself on the market. In addition to a variant known from the Prius, a drive system based on the same concept and supplemented by a 4-speed transmission is considered. This allows determination of the influence of increased mechanical complexity on Power-Split-DHT (PS-DHT).

\subsubsection{Power-Split DHT}

An important DHT concept with a high market share is the power-split concept introduced by Toyota in 1997. In this paper, the transmission structure of the 4th generation of Toyota's power-split concept, introduced in [2], is investigated. The drive train structure is shown in Figure 4.

The most important component of the transmission is a PGS. The ICE is connected to the planet carrier $\mathrm{C}$. One EM is connected to the sun gear $\mathrm{S}$ and one to the ring gear $\mathrm{R}$. The EM connected to the ring gear is connected to the driven axle via a reduction gear. Furthermore, a one-way clutch (OWC) is connected to the PGS so that the vehicle can be driven by both EM in electric driving. Without the OWC, EM1 would need to be controlled so that the planet carrier would be stationary to turn off the ICE. 


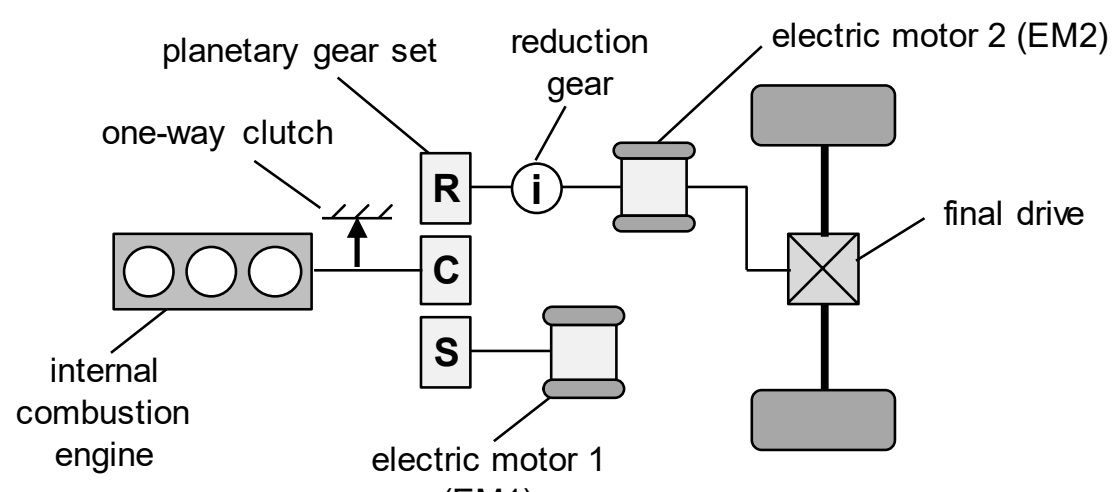

(EM1)

Figure 4. PS-DHT drive train topology with planetary gear set-R: ring gear, C: planet carrier, S: sun gear.

Apart from electric driving, the PS-DHT offers a hybrid mode with a continuously variable gear ratio. This can be realized by controlling the speed of EM1 so that the mode can also be called electronic continuously variable transmission (eCVT) mode. In this operating mode, the speed and torque of the ICE can be chosen freely.

The concept is comparatively simple regarding the mechanical effort. In addition to the planetary gear set, the relevant components include the OWC, the reduction gear for EM2 and the FD. This results in a mechanical effort of 4 .

\subsubsection{Power-Split DHT with 4-Speed Transmission}

The PS-DHT structure can be combined with a 4-speed transmission. A similar concept is introduced in [3,4]. Figure 5 shows a modified structure with an OWC between ICE and the planet carrier so that both EM can operate during electric driving. The 4-speed transmission is positioned between EM2 and the FD so that the speed of EM2 and the ring gear can be changed. Thus, it influences the power flow between the energy converters but does not add an output-split or compound-split operating mode. Instead, four input-split eCVT modes and four electric driving modes can be selected.

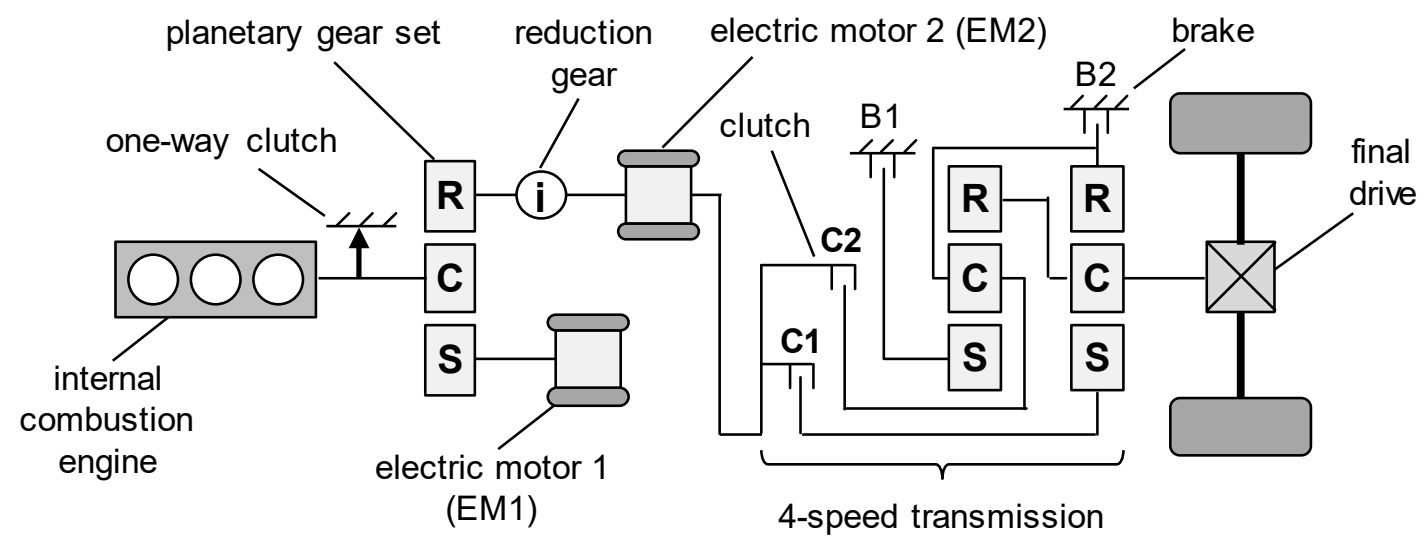

Figure 5. Drive train topology of PS-DHT enhanced by 4-speed transmission-R: ring gear, C: planet carrier, S: sun gear.

The 4-speed transmission consists of two PGS and four shifting elements. By controlling two clutches C1 and C2 and two brakes B1 and B2, four gear ratios can be selected. The shift matrix is shown in Table 2. 
Table 2. Shift matrix of PS-DHT with 4-speed transmission.

\begin{tabular}{cccccc}
\hline Operating Mode & OWC & B1 & B2 & C1 & C2 \\
\hline eCVT 1st & & & $\bullet$ & $\bullet$ & \\
eCVT 2nd & & $\bullet$ & & $\bullet$ & $\bullet$ \\
eCVT 3rd & & $\bullet$ & $\bullet$ & $\bullet$ & $\bullet$ \\
eCVT 4th & $\bullet$ & & & $\bullet$ & \\
electric 1st & $\bullet$ & $\bullet$ & & $\bullet$ \\
electric 2nd & $\bullet$ ectric 3rd & $\bullet$ & & & $\bullet$ \\
electric 4th & $\bullet$ & $\bullet$ & & & \\
\hline
\end{tabular}

Compared to the PS-DHT without the 4-speed transmission, the powertrain is more complex. The increased mechanical effort directly results from the 4-speed transmission. For the quantification of the mechanical effort, three PGS, 5 shifting elements and the FD must be taken into account. Thus, the mechanical complexity can be estimated with 9 .

\subsection{Multi-Mode DHT Concepts}

MM-DHT represent another category of hybrid transmission. They differ from PS-DHT since they do not only have an input-split eCVT mode in addition to electrical operation, but also offer additional functions. Depending on the structure of the concepts, several parallel or additional power-split modes, such as compound-split modes, can be implemented. This paper analyzes two concepts that are used in production vehicles. These include the concept from the second-generation Chevrolet Volt, introduced in [5] and the concept from the Cadillac CT6 PHEV, introduced in [6]. The influence of increased mechanical complexity compared to the basic concept can also be determined for the MM-DHT.

\subsubsection{Multi-Mode-DHT with two PGS}

Figure 6 shows the structure of the MM-DHT with two PGS and two active shifting elements. The concept shown, corresponds to the drive system of the Chevrolet Volt 2, see [5]. The ICE is connected to the ring gear of the first PGS and can drive the vehicle. The sun gears of the PGS are each connected to EM, while the planet carriers form a common output. The sun gear of the first PGS can be connected to the second one via a clutch C. In addition, the ring gear of the second PGS can be stationary, when brake B is actuated. With the PGS and three shifting elements, the concept enables two electric, two power-split, and one parallel operating mode. The corresponding shift matrix is shown in Table 3.

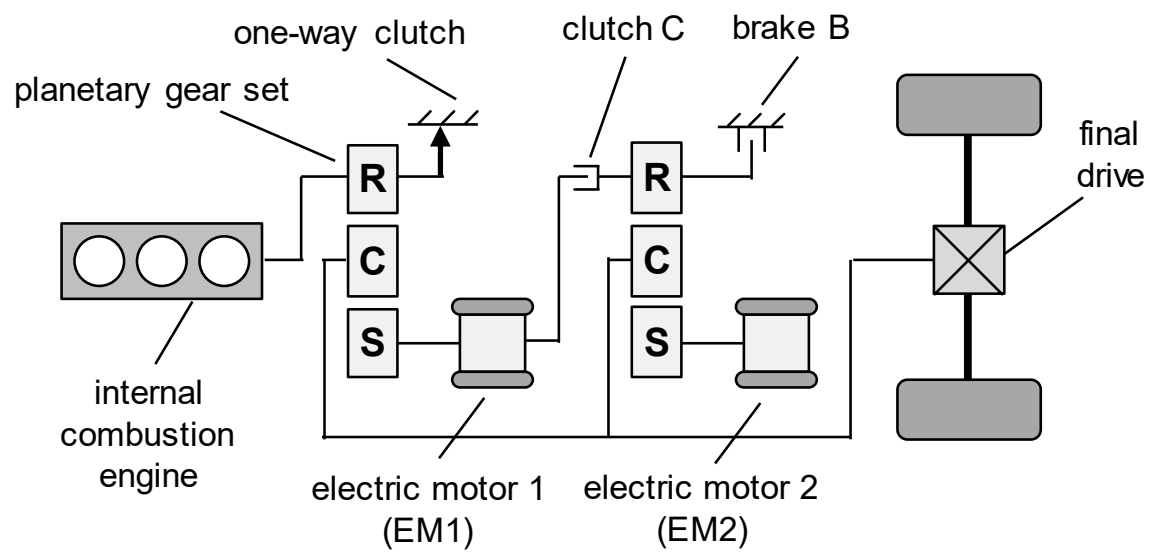

Figure 6. Drive train topology of MM-DHT with two planetary gear sets-R: ring gear, C: planet carrier, S: sun gear. 
Table 3. Shift matrix of MM-DHT with two PGS.

\begin{tabular}{cccc}
\hline Operating Mode & OWC & B & C \\
\hline eCVT 1st & & $\bullet$ & \\
parallel mode & & $\bullet$ & $\bullet$ \\
eCVT 2nd & & & $\bullet$ \\
electric 1st & $\bullet$ & $\bullet$ & \\
electric 2nd & & $\bullet$ & \\
\hline
\end{tabular}

In mode eCVT 1st there is an input-split power flow while in mode eCVT 2nd there is a compound-split power flow. The shift matrix in Table 3 shows that in mode electric 1st both EM can drive the vehicle, while in mode electric 2nd only EM2 can operate.

With the two PGS, three shifting elements and the axle drive, the mechanical effort for the concept can be quantified to 6 . This results in a complexity between the PS-DHT and the PS-DHT with 4 -speed transmission.

\subsubsection{Multi-Mode-DHT with three PGS}

An extension of the MM-DHT with two PGS is the MM-DHT with three PGS, see Figure 7. Up to the third PGS, the design is identical. The drive system is derived from the Cadillac CT6 PHEV, see [6]. The planet carrier of the first two PGS no longer forms the transmission output but is connected to the sun gear of the third PGS. The EM2 is connected to the ring gear of the third PGS via a clutch C2. The ring gear R is stationary when brake B2 is actuated. The output is the planet carrier of the third PGS.

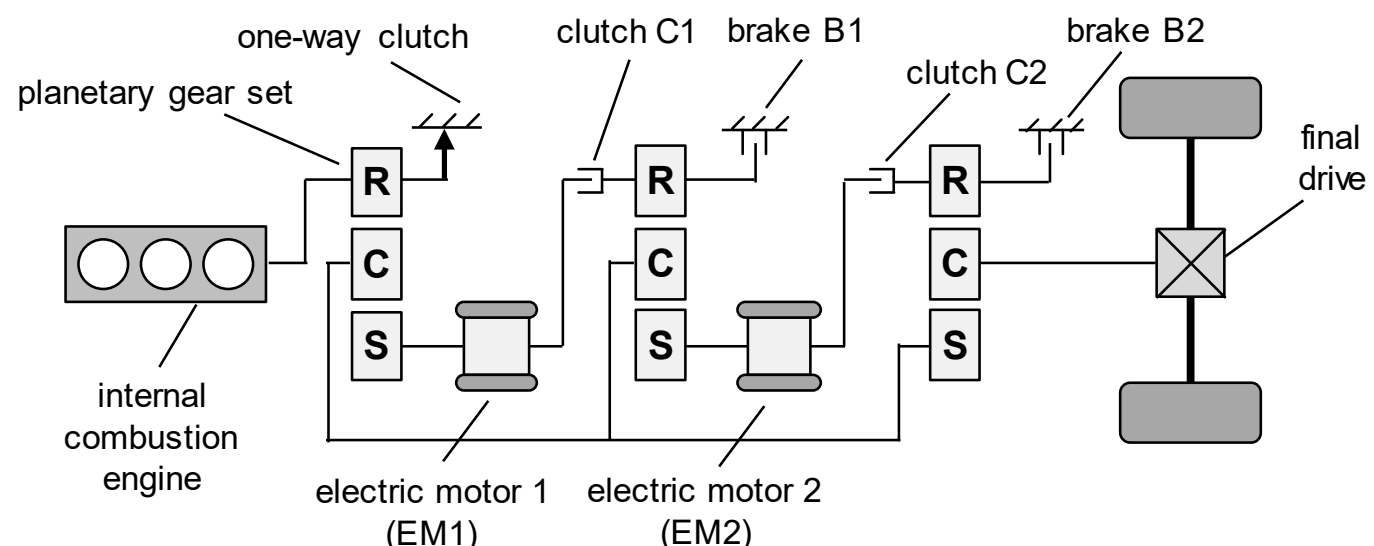

Figure 7. Drive train topology of MM-DHT with three planetary gear sets-R: ring gear, C: planet carrier, S: sun gear.

With the three PGS and five shifting elements, 11 operating modes can be implemented. These include four electrical operating modes in which either one or both EM can operate. In addition, four eCVT modes are available in which either an input-split or compound-split power transmission occurs. In addition, three parallel modes with a constant gear ratio between ICE and wheel can be selected. The shift matrix of the concept is shown in Table 4. 
Table 4. Shift matrix of MM-DHT with three PGS.

\begin{tabular}{cccccc}
\hline Operating Mode & OWC & B1 & B2 & C1 & C2 \\
\hline electric 1st & $\bullet$ & $\bullet$ & $\bullet$ & & \\
electric 2nd & $\bullet$ & & $\bullet$ & $\bullet$ & $\bullet$ \\
electric 3rd & $\bullet$ & & & & \\
electric 4th & $\bullet$ & $\bullet$ & $\bullet$ & & \\
eCVT 1st & & $\bullet$ & $\bullet$ & $\bullet$ \\
parallel 1st & & $\bullet$ & $\bullet$ & $\bullet$ & $\bullet$ \\
eCVT 2nd & & & & $\bullet$ & $\bullet$ \\
parallel 2nd & & & & $\bullet$ \\
eCVT 3rd & & $\bullet$ & & \\
parallel 3rd & & & & & \\
eCVT 4th & & & & & \\
\hline
\end{tabular}

Compared to the MM-DHT with two PGS, the MM-DHT with three PGS is more complex. An additional PGS and two additional shifting elements increase the mechanical complexity from 6 to 9 .

\section{Vehicle Parameters}

One aim of this paper is to show that there is a connection between the total electrical power required to meet the driving performance requirements and the mechanical effort of a concept. For this purpose, the three high-volume vehicle segments $C, D$, and $E$ are considered. For all concepts, equal driving performance requirements are defined within a segment. Table 5 shows the vehicle parameters of the three segments.

Table 5. Vehicle parameters for C, D, and E segment vehicle.

\begin{tabular}{ccccc}
\hline Parameter & Unit & C Segment & D Segment & E Segment \\
\hline mass & $\mathrm{kg}$ & 1600 & 1700 & 1900 \\
max. additional load & $\mathrm{kg}$ & 500 & 550 & 650 \\
frontal area & $\mathrm{m}^{2}$ & 2.2 & 2.2 & 2.35 \\
drag coefficient & - & 0.27 & 0.26 & 0.25 \\
rolling resistance coefficient & - & & $8 \times 10^{-3}$ & \\
residual braking force & $\mathrm{N}$ & 40 & 40 & 45 \\
wheelbase & $\mathrm{m}$ & 2.65 & 2.8 & 2.95 \\
weight distribution front to & $\%$ & $60: 40$ & $60: 40$ & $50: 50$ \\
rear, empty vehicle & & & $50: 50$ & $45: 55$ \\
weight distribution front to & $\%$ & $50: 50$ & 0.5 & \\
rear, loaded vehicle & $\mathrm{m}$ & 0.31 & 0.32 & 0.32 \\
center of gravity height & $\mathrm{m}$ & 100 & 150 & 225 \\
wheel radius & $\mathrm{kW}$ & front & front & rear \\
max. power of ICE & - & 10 & 10 & 10 \\
driven axle & $\mathrm{kWh}$ & & & \\
battery capacity & & & & \\
\hline
\end{tabular}

This paper examines concepts suitable for use in PHEV. For this reason, typical vehicle parameters are defined. The vehicle mass of the $C$ segment is assumed $1600 \mathrm{~kg}$. It increases to $1700 \mathrm{~kg}$ for the D segment and $1900 \mathrm{~kg}$ for the E segment. The maximum payload in the $\mathrm{C}$ segment is $500 \mathrm{~kg}$, 550 $\mathrm{kg}$ in the D segment and $650 \mathrm{~kg}$ in the E segment. The wheelbase increases from $2.65 \mathrm{~m}$ for the C segment over $2.8 \mathrm{~m}$ in the D segment to $2.95 \mathrm{~m}$ in the E segment. Another important parameter is the center of gravity of the vehicles. In the unloaded case, the vehicles in the $C$ and $D$ segments are front-loaded with a weight distribution of 60:40, while the vehicle in the E segment has a balanced weight distribution. If the vehicles are loaded by their maximum payload, the center of gravity of the vehicle changes. In case of the $C$ and $D$ segments, a balanced weight distribution results. The vehicle of the E segment has a weight distribution of 45:55 when fully loaded. 
Another important difference between the segments is the maximum power of the ICE. For the $C$ segment vehicle, a naturally aspirated engine with a maximum output of $100 \mathrm{~kW}$ is considered. The D segment is based on a supercharged engine with $150 \mathrm{~kW}$, while the $\mathrm{E}$ segment is based on a supercharged engine with a maximum power of $225 \mathrm{~kW}$. For all concepts within a segment, the equal combustion engine is used to determine the effects of a certain drive train structure on important properties such as performance and efficiency. It should be noted that it is neglected that the ICE can be optimized for some concepts.

As the reference vehicle is a PHEV and there are no requirements for the electrical range, the battery capacity is $10 \mathrm{kWh}$.

The driven axle also influences the driving performance requirements. In the $\mathrm{C}$ and $\mathrm{D}$ segments, the vehicles are front-wheel-drive (FWD) and in the E segment rear-wheel-drive (RWD).

The residual brake force describes an additional driving resistance resulting from residual braking torques of the wheel brake and wheel bearing friction.

\section{Driving Performance Requirements}

In hybrid drive trains, multiple energy storages and energy converters are present. Depending on their interaction, the power available on the wheel may vary. Therefore, several drive train states should be taken into account when defining driving performance requirements. According to [1], there can be several ways in which the energy can be provided by either the battery or the ICE in case of HEV. In this article, three corresponding states are taken into account when defining driving performance requirements. In hybrid mode, the battery can either be charged by the ICE or provide additional power to drive the vehicle. In addition to hybrid operation, it is also possible to define driving performance requirements for electrical operation. The power available at the wheel varies as a result of the power provided by the energy converters. This is shown in Figure 8 for different drive states.

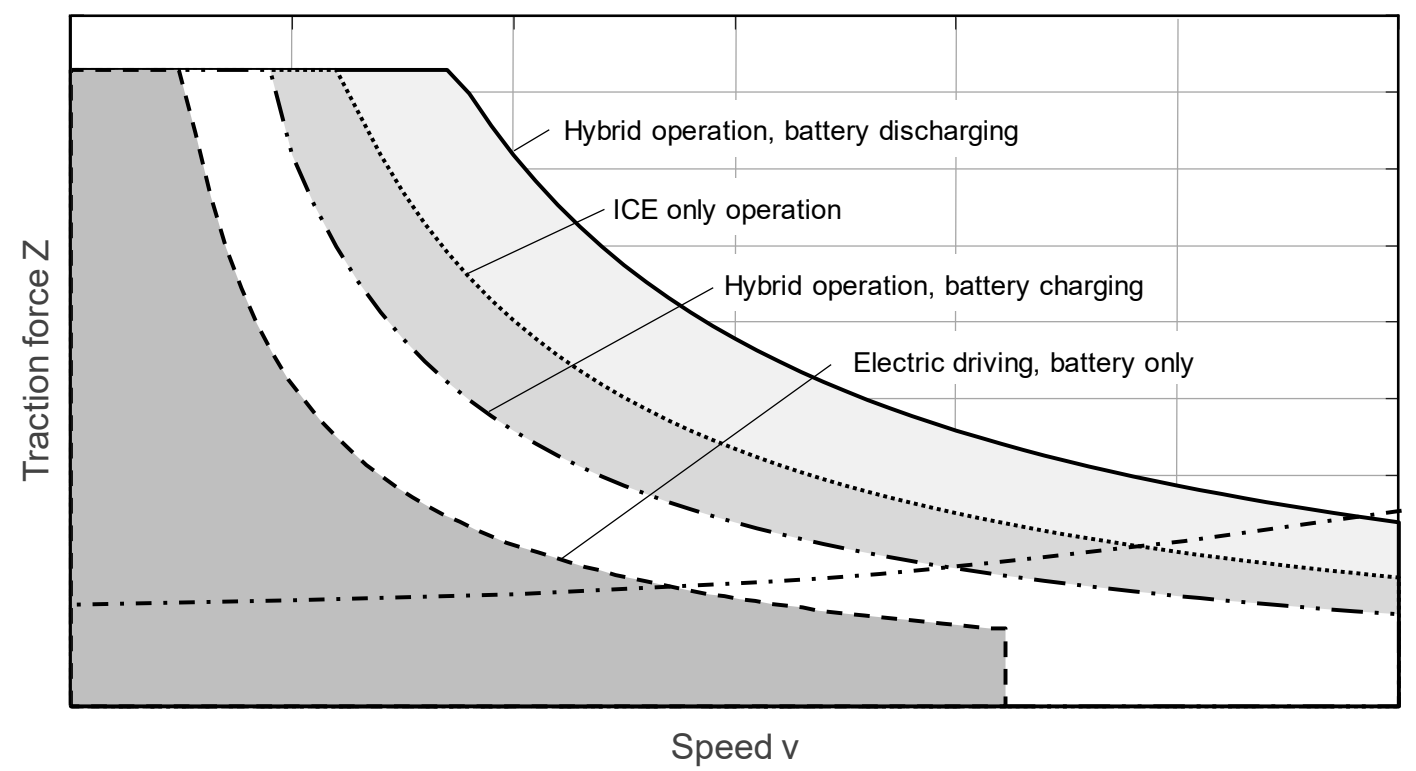

Figure 8. Traction force at wheel level for different drive train states.

The number of defined driving performance requirements should be as low as possible to be able to consider many possible variants within a parameter variation. At the same time, it needs to be ensured that a sufficient number of driving situations, i.e., tractive forces at a certain speed, are taken into account. Therefore, there is a conflict between the number of requirements and the required computation time. In this paper, driving performance requirements for different drive states are defined at a small number of vehicle speeds, so that there are several requirements per drive state 
in order to keep the computing time as short as possible. The requirements are distributed over the entire speed range of the vehicle to ensure that the resulting delivery maps contain realistic driving situations. It should be noted that the specified driving performance requirements represent minimum requirements, which all variants of each concept must meet. The requirements within one segment are the same for all concepts to enable a fair comparison. In addition, the designed concepts may exceed the minimum requirements. Table 6 summarizes the requirements.

Table 6. Drive train state specific driving performance requirements for C, D, and E segment vehicle.

\begin{tabular}{|c|c|c|c|c|c|}
\hline Drive Train State & Parameter & Unit & C Segment & D Segment & E Segment \\
\hline \multirow{3}{*}{$\begin{array}{l}\text { Hybrid operation, } \\
\text { battery discharging }\end{array}$} & $\begin{array}{l}\text { traction force limit } \\
\text { loaded vehicle } \\
\mathrm{v} \leq 10 \mathrm{~km} / \mathrm{h}\end{array}$ & - & yes & yes & Yes \\
\hline & $\begin{array}{l}\text { power at ICE level due to } \\
\text { boosting at } \mathrm{v}=80 \mathrm{~km} / \mathrm{h}\end{array}$ & $\mathrm{kW}$ & $100+40$ & $150+50$ & $225+60$ \\
\hline & max. speed & $\mathrm{km} / \mathrm{h}$ & 200 & 225 & 250 \\
\hline \multirow{3}{*}{$\begin{array}{l}\text { Hybrid operation, } \\
\text { battery charging }\end{array}$} & $\begin{array}{l}\text { traction force limit } \\
\text { loaded vehicle } \\
\text { v } \leq 10 \mathrm{~km} / \mathrm{h}\end{array}$ & - & yes & yes & yes \\
\hline & $\begin{array}{l}\text { power at ICE level due to } \\
\text { charging at } \mathrm{v}=80 \mathrm{~km} / \mathrm{h}\end{array}$ & $\mathrm{kW}$ & 100-10 & 150-10 & $225-20$ \\
\hline & max. speed & $\mathrm{km} / \mathrm{h}$ & 200 & 225 & 250 \\
\hline \multirow[t]{3}{*}{ Electric driving } & $\begin{array}{l}\text { traction force limit } \\
\text { loaded vehicle } \\
\mathrm{v} \leq 10 \mathrm{~km} / \mathrm{h}\end{array}$ & - & yes & yes & yes \\
\hline & $\mathrm{t}_{60-100} / \mathrm{a}(\mathrm{v}=80 \mathrm{~km} / \mathrm{h})$ & $\mathrm{s}$ & 6 & 5 & 4 \\
\hline & max. speed & $\mathrm{km} / \mathrm{h}$ & 135 & 145 & 160 \\
\hline
\end{tabular}

It should be noted that the requirements in hybrid operation for charging or discharging the battery refer to the transmission input. The requirement at wheel level is calculated for a powertrain efficiency of $90 \%$.

In hybrid operation, when the battery is discharged, all variants, irrespective of the segment, must reach the traction force limit of the fully loaded vehicle in a speed range up to at least $10 \mathrm{~km} / \mathrm{h}$ to ensure sufficient gradeability. In all DHT concepts considered, one of the EM is responsible for providing sufficient torque on wheel level until the traction force limit is reached. Since EM can deliver their maximum torque over a wider speed range, there are delivery maps in which the traction force limit is reached at speeds higher than $10 \mathrm{~km} / \mathrm{h}$. It is, therefore, sufficient to require the concepts to reach the traction force limit at least in a speed range between $0 \mathrm{~km} / \mathrm{h}$ and $10 \mathrm{~km} / \mathrm{h}$.

For the medium speed range at $80 \mathrm{~km} / \mathrm{h}$, it is required that the variants must achieve a certain traction force. To be able to quantify this traction force, ICE operation without discharging the battery is the starting point. For hybrid operation with battery discharge, a boost power is defined that must be applied in addition to the maximum ICE power. For vehicles in the $C$ segment, $140 \mathrm{~kW}$ of power must be available at the transmission input. This power results from a nominal ICE power of $100 \mathrm{~kW}$ in combination with a boost power of $40 \mathrm{~kW}$. Taking into account a drive train efficiency of $90 \%$, the necessary power or traction force can thus be calculated at wheel level. For the other segments, there is a differentiation due to a higher ICE maximum power as well as a higher boost power.

To define a driving performance requirement in the higher speed range and to limit the demand map of the vehicles, the variants in the $C$ segment are required to achieve a maximum speed of 200 $\mathrm{km} / \mathrm{h}$ in hybrid operation when the battery is discharged. In the D and E segment, the maximum speed is $225 \mathrm{~km} / \mathrm{h}$ and $250 \mathrm{~km} / \mathrm{h}$, respectively.

In addition to discharging the battery, hybrid operation while charging the battery is a relevant drive train state since less power is available on the wheel. The approach to determine the corresponding tractive force is identical to hybrid operation while discharging the battery. The only difference is that no additional power is provided, but is reserved for charging the battery. In the C and D segment, a reserve of $10 \mathrm{~kW}$ is defined, while in the E segment the maximum ICE power is reduced by $20 \mathrm{~kW}$. 
This does not mean that the battery is charged with the specified power, but takes into account the fact that some concepts in eCVT mode or serial mode require the battery to be charged in a certain speed range, resulting in increased losses. In the driving performance calculation of the simulation model, see Section 5, no charging power of the battery is specified. The model only ensures a negative battery power. In hybrid operation while charging the battery, the requirements are identical to the drive train state in which the battery is discharged.

It is characteristic for PHEV that they can achieve higher distances in electrical operation due to their battery capacity. Compared to mild or full hybrids, the driving performance requirements for electrical operation are therefore becoming more important. For the vehicles of all segments, it is specified that they must reach the traction force limit when the vehicle is fully loaded. The only exception is the P1P4 topology with an electric all-wheel drive. In electric operation, this vehicle would have RWD and would therefore offer a considerably higher traction potential than vehicles with FWD in the $C$ and $D$ segment. This would lead to significantly higher tractive forces required for electrical operation and would contradict a fair comparison of concepts. Therefore, the variants of the P1P4 topology are required to reach the traction force limit of a FWD vehicle in electrical operation.

To define a driving performance requirement in the medium speed range, an acceleration time from $60 \mathrm{~km} / \mathrm{h}$ to $100 \mathrm{~km} / \mathrm{h}$ is used as a distinguishing feature. It is assumed that below 60 $\mathrm{km} / \mathrm{h}$ there are minor differences regarding the acceleration time between different vehicle segments. Therefore, the acceleration time between $60 \mathrm{~km} / \mathrm{h}$ and $100 \mathrm{~km} / \mathrm{h}$ can be used to determine an average longitudinal acceleration at $80 \mathrm{~km} / \mathrm{h}$. This acceleration requirement directly corresponds to a traction force requirement. It should be noted that the acceleration time only servers as an orientation for deriving an average acceleration or traction force and does not represent a requirement to be achieved.

For the variants in the $\mathrm{C}$ segment, the acceleration time is $6 \mathrm{~s}$, in the $\mathrm{D}$ segment it declines to $5 \mathrm{~s}$ and in the E segment to $4 \mathrm{~s}$.

In electrical operation, the requirement for the maximum vehicle speed in the $C$ segment is based on the speed profile of the Worldwide harmonized Light Duty Test Cycle (WLTC). To ensure that the cycle can be fully completed in electrical operation, a maximum speed of $135 \mathrm{~km} / \mathrm{h}$ must be reached. So that vehicles in higher segments differ from those of the $C$ segment, the maximum speed in the $\mathrm{D}$ segment is $145 \mathrm{~km} / \mathrm{h}$ and $160 \mathrm{~km} / \mathrm{h}$ in the E segment.

\section{Simulation Model}

A modular simulation model developed by [1] makes a significant contribution to the results in this paper. The structure and the essential functionality are explained in this section. Explanations that are more detailed can be found in [1].

An essential feature of the simulation model is that the calculation of operating points and the efficiency of drive concepts is coupled with a driving performance calculation. Based on the possible driving performance, i.e., the delivery maps of a drive train concept, an efficiency calculation can be carried out in any speed profile. Therefore, the model can be used on the one hand for estimating driving performance and thus for determining appropriate drive train variants. On the other hand, it enables the simulation of almost any drive topology in cycle or customer operation.

A schematic structure of the model is shown in Figure 9. Based on driving resistances due to the vehicle parameters, operating points of the drive train components and energy converters are calculated backwards through the drive train. Based on efficiency maps of the energy converters, the vehicle's energy consumption can be determined. 


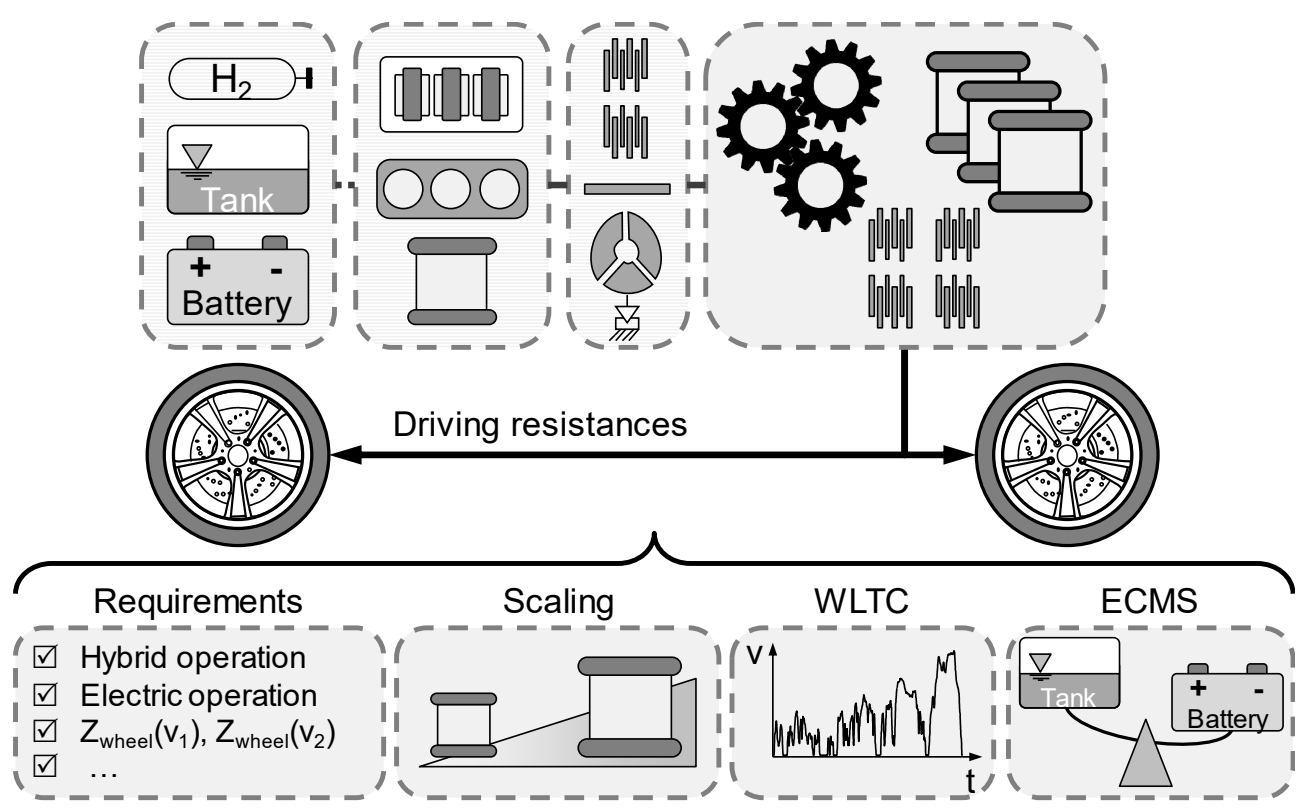

Figure 9. Schematic structure of the modular simulation model developed and inspired by [1].

A central component of the model is a modular transmission calculation, which calculates the associated speeds and torques within the transmission based on all possible operating points of the energy converters. The calculation has a modular structure so that a wide variety of transmission concepts can be calculated within a short time with little parameterization effort. In addition to the calculation of conventional transmissions, it is possible to simulate concepts in which several EM are integrated into the transmission. This feature is particularly necessary for the simulation of complex DHT with several EM.

To calculate the transmission losses, two approaches can be selected. In addition to a simplified approach, which describes the efficiency depending on the output power of the transmission, mode-specific efficiency maps can be implemented. In this paper, however, the simplified approach is used to reduce the calculation time.

Two primary energy converters can be positioned outside the transmission. They can be connected to the transmission module via a launch element. The primary energy converters include ICE, fuel cells and EM.

In addition to the modules for calculating driving performance and efficiency, driving performance requirements can be defined. With the help of the performance calculation, it is then possible to determine which variants of a drive train concept can meet the requirements. Within the scope of parameter variations, fractional or full factorial scaling plans can be considered.

To select the most efficient operating points, two operation strategy approaches are implemented. In addition to a globally optimal control strategy, presented in $[7,8]$, there is a locally optimal operating strategy based on the equivalent consumption minimization strategy (ECMS) $[9,10]$. This local optimal approach is used in the following considerations.

\section{Dimensioning of Electric Motors}

The simulation tool described in Section 5 is used to determine powertrain designs that meet the requirements described in Section 4. For each concept considered in this paper, a range of design parameters is defined. These include upper limits for the maximum power of the EM. Furthermore, intervals are defined in which ratios of PGS, the FD or the reduction gears are varied.

The driving performance calculation allows determination of which variants meet the requirements. The variants whose total EM power is minimal are of particular importance. Designs with a significantly higher total power are of less importance as they are oversized regarding the requirements. Based on 
the driving performance requirements and the drive train concepts, the above-mentioned relationship between mechanical and electrical effort can be confirmed. Furthermore, the investigations allow drawing of conclusions about the suitability of concepts in different vehicle segments.

Figure 10 shows the minimum required power of the EM which is necessary to meet the requirements. Furthermore, the mechanical effort quantified by a figure, see Section 2, is compared.

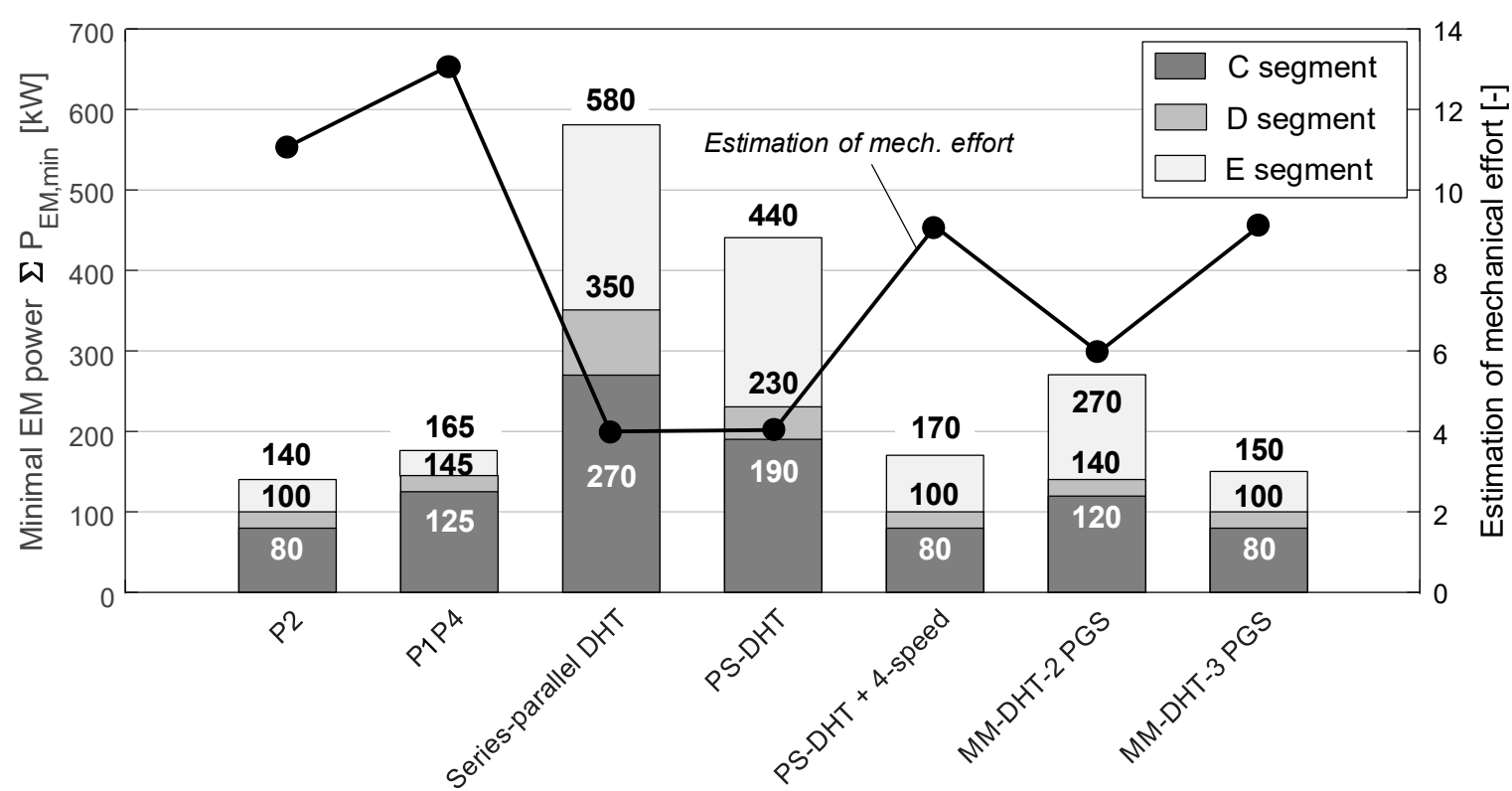

Figure 10. Concept-specific minimal required EM power to meet the driving performance requirements in the C, D, and E segment in comparison with the estimated mechanical effort of the concepts.

\subsection{Analysis of Total Necessary EM Power for C Segment Requirements}

In the $\mathrm{C}$ segment, the minimum required EM power for the P2 HEV is $80 \mathrm{~kW}$. Due to the eight gears of the DCT, the necessary EM power does not result from the requirements in hybrid operation, but is due to the requirement in electric operation at $80 \mathrm{~km} / \mathrm{h}$. Reaching the traction force limit at full load can also be ruled out as a cause since the first gear already enables a high gear ratio. A power of $80 \mathrm{~kW}$ is therefore not required to reach the traction force limit.

The total necessary electrical power of the P1P4 HEV increases to $125 \mathrm{~kW}$. This consists of $15 \mathrm{~kW}$ for the P1-EM and $110 \mathrm{~kW}$ for the P4-EM. It should be noted that a power of $15 \mathrm{~kW}$ has been defined for the P1-EM for all variants of the P1P4 and in all segments. Compared to the P2 HEV, the higher power of the EM results from the fact that there is only a constant gear ratio between EM and wheel and that the EM can only be separated from the drive train when its speed limit is reached. To reach the maximum speed in electrical operation, the reduction gear ratio for the EM must not exceed a certain value. The P4-EM must therefore provide a sufficiently high torque to allow the vehicle to reach the traction force limit when launching with full load.

The total necessary electrical power in case of the series-parallel DHT increases considerably compared to the add-on concepts. In total, $270 \mathrm{~kW}$ of electrical power must be installed in the drive train to meet the requirements in the $\mathrm{C}$ segment. This high power demand results from the requirements in hybrid operation when charging the battery. In this case, the concept offers two operating modes. In parallel mode, the ICE can only provide the required wheel power in a certain speed range since there is a constant gear ratio between ICE and wheel. In case the ICE cannot provide enough power to meet the requirement, the serial mode needs to be selected. In this mode, the ICE provides power to drive the vehicle and EM1, see Figure 10, needs to have a similarly high power to be able to transmit the power electrically so that EM2 can drive the vehicle. EM2 therefore at least needs to have a power which is similar to the power of EM1. 
To reach the traction force limit, EM2 must provide a high torque during charging of the battery in hybrid operation and during electric driving. There is a similar correlation as with the P4-EM, so that a high torque requirement results in a correspondingly high power for EM2.

The PS-DHT requires a lower total EM power than the series-parallel concept. In the $\mathrm{C}$ segment, a total power of $190 \mathrm{~kW}$ is required. In this case, EM1 needs to have a power of $60 \mathrm{~kW}$ and EM2 is required to provide $130 \mathrm{~kW}$. The significantly higher power of EM2 is needed since the traction force limit must be reached in electric and hybrid operation. Compared to the PS-DHT concept from [4], the minimum required power of EM1 is higher, since high requirements have been defined for hybrid operation with simultaneous charging of the battery.

If a 4-speed transmission is added to the PS-DHT, the minimum power required is reduced to a total amount of $80 \mathrm{~kW}$. This results in an electrical effort comparable to the P2 HEV. The 4-speed transmission, see Figure 5, allows adjustment of the operating point of the EM2 and the speed of the ring gear. This reduces the torque required for EM2 to reach the traction force limit. Furthermore, the speed of the ring gear influences the power flow in power-split mode, so that the power of EM1 can also be reduced.

The required power of the EM of the MM-DHT with two PGS increases to $120 \mathrm{~kW}$. Compared to the PS-DHT, the power is $70 \mathrm{~kW}$ lower. Due to a higher number of possible operating modes, including two power-split eCVT modes and a parallel mode, more degrees of freedom are available in hybrid operation when the battery is discharged.

The MM-DHT with three PGS requires as much electrical power as the P2 HEV and the PS-DHT with 4-speed transmission. By increasing the number of available operating modes, including four eCVT modes and three parallel modes, it is possible to install a lower total electrical power than in the similar concept with two PGS.

It can be concluded that with the EM maps used and the requirements applied, the minimum electrical power required in the $\mathrm{C}$ segment is $80 \mathrm{~kW}$.

\subsection{Analysis of Total Necessary EM Power for D and E Segment Requirements}

Due to the higher requirements in the $\mathrm{D}$ segment, the total electrical power required for all concepts increases. However, a comparison between the concepts shows that the increase differs among the concepts. For example, the P2 HEV's and the P1P4 HEV's output increases by $20 \mathrm{~kW}$. It should be noted that the increase for the P1P4 HEV only affects the P4-EM, as it was specified that the P1-EM should have an output of $15 \mathrm{~kW}$ in all segments.

A higher increase results in case of the series-parallel DHT. For this concept, the electrical effort increases by $80 \mathrm{~kW}$ to $350 \mathrm{~kW}$. This corresponds to an increase of approx. $29.6 \%$. On the one hand, the increase in power can be explained by the higher traction force limit due to the vehicle parameters. On the other hand, the increased electrical power requirement results from hybrid operation when the battery is charged. In this case, only the ICE is available as a power source. The power provided by the ICE is received by EM1, see Figure 3. As a result of this dependency, the power of EM1 is directly dependent on the power of the ICE.

The power of EM1 is also dependent on the power of the ICE in case of the PS-DHT. When charging the battery with low power, however, a portion of the power provided by the ICE can be transferred mechanically to the wheel. Therefore, the EM1 of the PS-DHT does not have to receive the maximum power of the ICE. As a result, the total electrical power required for the PS-DHT increases by approx. $21 \%$ compared to the $C$ segment. The total required power of the EM is $230 \mathrm{~kW}$.

The 4-speed transmission allows adjustment of the operating point of EM2 and the speed of the ring gear. This reduces the total electrical power required compared to the PS-DHT without a multi-speed transmission. Therefore, in hybrid operation with discharged battery less power must be transmitted via the EM in the case of split power mode, which directly reduces the power requirement of the EM. As a result of this, an electrical effort of $100 \mathrm{~kW}$ is required in the D segment. 
A total electrical power demand of $140 \mathrm{~kW}$ results for the MM-DHT with two PGS in the D segment. Compared to the $C$ segment the increase of approx. $16.7 \%$ is lower. In comparison to the PS-DHT, less electrical power is required because two eCVT modes and one parallel mode are available.

If the MM-DHT with two PGS is supplemented by an additional PGS, $100 \mathrm{~kW}$ of electrical power are required in the $\mathrm{D}$ segment. As in the $\mathrm{C}$ segment, this concept has a similar electrical effort as the P2 HEV and the PS-DHT with 4-speed transmission.

In the E segment, the total electrical power required for all concepts increases as a result of increased requirements. The P2 HEV, PS-DHT with 4-speed transmission, and MM-DHT with three PGS continue to have the lowest electrical effort. However, there are slight differences between these concepts in the E segment. The total necessary EM power for the series-parallel DHT increases to $580 \mathrm{~kW}$. The relative increase is even higher for the PS-DHT, but with $440 \mathrm{~kW}$ less electrical power is required in total. Compared to the PS-DHT, the required EM power of the MM-DHT with two PGS in the E segment increases to a comparatively lower value of $170 \mathrm{~kW}$.

\subsection{Relationship between Mechanical and Electrical Effort}

In addition to the total electrical power required, Figure 10 shows an estimate of the mechanical effort for the considered concepts. If this is compared with the required electrical power, a correlation between electrical and mechanical effort is shown.

The results show that the concepts with high mechanical effort require a comparatively low electrical effort in all vehicle segments. The P2 hybrid has a mechanical effort of 11 and is the concept with the second highest mechanical complexity according to the estimation used in this paper. In all segments, the P2 HEV requires the lowest total electrical power.

Due to the additionally driven rear axle, the mechanical effort of the P1P4 HEV rises to 13. Compared to the $\mathrm{P} 2 \mathrm{HEV}$, a higher overall EM power is required in all segments. Therefore, no linear relationship between the mechanical effort and the electric effort can be shown. However, a clear trend can be identified based on the results. For future considerations, however, a different parameter should be used to estimate the mechanical effort to be able to describe a clearer relationship.

The PS-DHT with 4-speed transmission and the MM-DHT with three PGS also are concepts with a high mechanical effort. Each of these concepts is a mechanically more complex variant of a basic concept. In the $C$ and D segment, they have a similarly low necessary EM power as the P2 HEV, but with lower mechanical effort. Although there are differences in the E segment, the overall EM power is significantly lower compared to the other concepts with lower mechanical complexity.

If the mechanical effort of the concepts is significantly reduced, the required power of the EM increases significantly. Both the series-parallel DHT and the PS-DHT have a comparatively simple design with a mechanical effort of 4 . At the same time, these concepts require the highest EM power compared to the other concepts. The difference is particularly high in the E segment.

\subsection{Application of Powertrain Concepts in Different Vehicle Segments}

Based on the results in Figure 10, it can be concluded which concepts should be used in which vehicle segment. For this purpose, the ratio of electrical and mechanical effort can provide a helpful orientation. It should be noted that the assessment of the suitability of drive train concepts in different segments depends on the underlying requirements to a considerable extent. For example, a reduction of the requirements in hybrid operation while charging the battery would lead to significantly reduced EM power. Especially concepts with low mechanical effort would benefit from this. Nevertheless, it can be expected that mechanically complex concepts would still have the lowest power demand for electrical power.

However, based on the driving performance requirements in this paper, the use of the concepts for segments C, D, and E can be assessed.

The E segment shows considerable differences between the total electrical power required by the concepts. The largest difference with $440 \mathrm{~kW}$ is between the P2 HEV and the series-parallel DHT. The 
difference between P2 HEV and PS-DHT is $300 \mathrm{~kW}$. In comparison to the MM-DHT with two PGS, the difference decreases to $130 \mathrm{~kW}$. This leads to the conclusion that the series-parallel DHT as well as the PS-DHT in the E segment do not represent the most favorable solutions. For the MM-DHT with two PGS it is shown that the electrical effort can be reduced considerably compared to concepts with higher complexity. Thus, the required power output decreases by $120 \mathrm{~kW}$ in case of the MM-DHT with three PGS and by $100 \mathrm{~kW}$ in case of the PS-DHT with 4-speed transmission if the mechanical effort is increased by 3. Therefore, it can be concluded that the MM-DHT with two PGS should not be used in the E segment either. Instead, concepts with a mechanical complexity of 9 or higher represent promising solutions.

In the D segment, the series-parallel DHT as well as the PS-DHT require significantly lower EM power compared to the E segment. However, regarding a good compromise between electrical and mechanical effort, other concepts lead to a better solution. Compared to the PS-DHT, the electrical effort of the MM-DHT with two PGS is reduced by almost $100 \mathrm{~kW}$ with a moderate increase of the mechanical complexity. This leads to the conclusion that the MM-DHT with two PGS is a better concept than the PS-DHT and the series-parallel DHT in the D segment. Concepts that are mechanically more complex have a lower electrical power requirement. To be able to assess the applicability with sufficient quality in these cases, further criteria such as costs or required installation space need to be taken into consideration.

Compared to the D segment, the difference between the series-parallel DHT and the PS-DHT is similarly large in the $\mathrm{C}$ segment. The difference in the $\mathrm{D}$ segment between the series-parallel DHT compared to the $\mathrm{P} 2$ hybrid is about $338 \%$, while in the C segment it is $350 \%$. For PS-DHT, there are relative differences of $230 \%$ in the D segment and about $238 \%$ in the $\mathrm{C}$ segment. Although the difference between the concepts is similar, the absolute required power decreases, so that the series-parallel DHT should not be excluded from application in the $C$ segment. However, it becomes apparent that the PS-DHT as well as the MM-DHT with two PGS represent a better compromise between mechanical and electrical effort. For the mechanically more complex concepts, less clear statements can be made, since although a low total electrical power is required, criteria such as cost, scalability, or modularity play an important role and are not considered in this paper.

\section{Benchmark Analysis}

The approach described in Section 6 allows identification of the minimal required EM power which needs to be installed in the considered concepts to meet the performance requirements. Furthermore, the simulation model provides requirement-compliant transmission ratios, which are varied within a parameter variation. The gear ratios include the FD ratio for the P2 and P1P4 hybrids. In the case of the series-parallel DHT, the gear ratios of the reduction gear for EM2 and the FD are varied. In case of the PS-DHT and MM-DHT concepts, the PGS ratios, the gear ratio of the FD and, if available, the gear ratio of the reduction gear for EM2 are varied.

For the concepts considered in this paper, the parameter variations result in a high number of different drive train variants. These can differ from each other in terms of driving performance and efficiency. To be able to make a statement which concepts and which dimensioning represent promising solutions, the concepts are compared with each other with regard to their driving performance in hybrid operation and in electric operation as well as their efficiency in charge sustaining operation.

The analysis of the minimum required EM power shows that all considered concepts require a low electrical effort in the $C$ segment compared to higher segments. Additionally, the differences between the concepts are smaller than in the D or E segment. Therefore, the question arises whether a high mechanical effort should be realized in the $C$ segment to reduce the required electrical power. By comparing the driving performance and efficiency, it can be analyzed whether a high mechanical complexity results in advantages regarding driving performance and efficiency. Furthermore, the results can indicate whether concepts with high electrical effort should not be used in the $C$ segment. For these reasons, the following considerations are limited to the results of the $\mathrm{C}$ segment. 
To compare the efficiency of the concepts, the $\mathrm{CO}_{2}$ emissions in the WLTC for charge sustaining operation are calculated. During charge sustaining operation, it is ensured that the state of charge (SOC) of the battery is almost identical at the beginning and end of the cycle to allow a fair comparison of the concepts. To evaluate the driving performance, the acceleration times from $0 \mathrm{~km} / \mathrm{h}$ to $100 \mathrm{~km} / \mathrm{h}$ in hybrid as well as in electric operation are calculated. For these calculations, the simulation model presented in Section 5 is used. A locally optimal operating strategy based on the ECMS is used.

Since it can be assumed that realistic drive trains are not over-dimensioned with regard to their driving performance requirements, variants that are in the range of the minimum required total electrical power are selected for the concept comparison. Table 7 shows the selected EM power for all concepts.

Table 7. EM-specific and total EM power selected for $C$ segment drive train concepts.

\begin{tabular}{cccc}
\hline Drive Train Concept & $\mathbf{P}_{\text {EM1 }}(\mathbf{k W})$ & $\mathbf{P}_{\text {EM2 }} \mathbf{( k W )}$ & Total EM Power $(\mathbf{k W})$ \\
\hline P2 HEV & 80 & - & 80 \\
\hline P1P4 HEV & 15 & $110-120$ & $125-135$ \\
\hline Series-parallel DHT & 110 & $160-170$ & $270-280$ \\
\hline PS-DHT & $60-70$ & $130-140$ & $190-210$ \\
\hline PS-DHT + 4-speed transmission & 40 & 40 & 80 \\
\hline MM-DHT - 2 PGS & $50-60$ & $80-90$ & $80-90$ \\
\hline MM-DHT - 3 PGS & 40 & $40-50$ &
\end{tabular}

In addition to the driving performance and the efficiency of the concepts, this article also evaluates the conversion quality defined by [11]. The concepts are compared regarding this benchmark parameter. Based on these results, important concept-specific properties are identified.

\subsection{Analysis of Efficiency in WLTC and Driving Performance}

In this section, the concepts in the $\mathrm{C}$ segment are compared regarding their efficiency in charge sustaining operation in the WLTC as well as their hybrid and electric driving performance. In hybrid operation, a distinction is made between driving performance with fully charged and discharged battery. In this case, the SOC-neutral $\mathrm{CO}_{2}$ emissions are not affected by the battery's state of charge, since the simulation ensures a SOC of $50 \%$ at the beginning and end of the cycle.

7.1.1. Evaluation of Efficiency and Driving Performance in Hybrid Driving while Discharging the Battery

The simulation results in case the battery can be discharged are shown in Figure 11. It should be noted that the emissions in charge sustaining mode do not correspond to the combined $\mathrm{CO}_{2}$ emissions, since the electrical energy consumption must also be analyzed and weighted to determine the combined $\mathrm{CO}_{2}$ emissions.

The P2 HEV variants achieve acceleration times of approx. $6.4 \mathrm{~s}$ from $0 \mathrm{~km} / \mathrm{h}$ to $100 \mathrm{~km} / \mathrm{h}$ in hybrid operation while the battery is discharged. Furthermore, $\mathrm{CO}_{2}$ emissions of about $111 \mathrm{~g} / \mathrm{km}$ to $111.6 \mathrm{~g} / \mathrm{km}$ are achieved. Compared to the other concepts, the P2 HEV variants achieve both good acceleration times and low $\mathrm{CO}_{2}$ emissions.

As a result of electrifying the rear axle with a P4-EM with an output of $110 \mathrm{~kW}$ or $120 \mathrm{~kW}$, considerably lower acceleration times can be achieved due to the electric all-wheel drive. Depending on the power of the EM on the rear axle, the variants achieve acceleration times in a range between 4.1 $\mathrm{s}$ and $4.4 \mathrm{~s}$. Thus, the difference compared to the P2 HEV is about $2 \mathrm{~s}$ or $2.3 \mathrm{~s}$. It should be noted that the simulation model allows a serial power flow in case of full-load acceleration for the P1P4 hybrid. If 
the front axle of the vehicle reaches the traction force limit, the P1-EM can transmit excess torque to the P4-EM positioned on the rear axle.

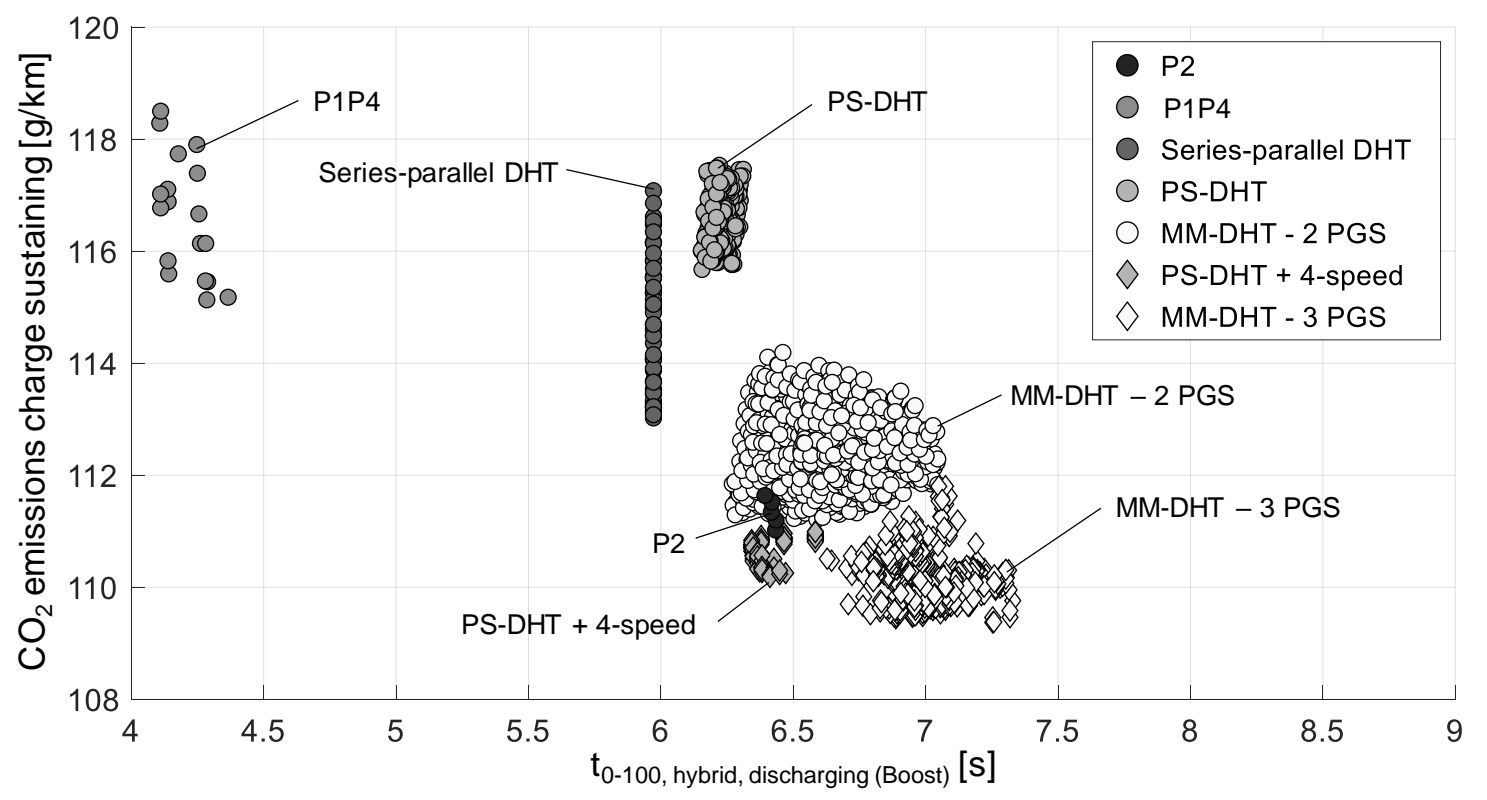

Figure 11. Comparison of charge sustaining efficiency in WLTC and hybrid driving performance for drive train concepts compliant with $\mathrm{C}$ segment requirements.

In contrast to the $\mathrm{P} 2 \mathrm{HEV}$, however, the $\mathrm{CO}_{2}$ emissions of the variants of the P1P4 concept are significantly higher. Depending on the dimensioning, the emissions range between $115 \mathrm{~g} / \mathrm{km}$ and 118.5 $\mathrm{g} / \mathrm{km}$. The higher $\mathrm{CO}_{2}$ emissions result from the fact that the P4-EM, which can be used during electric operation, is connected to the wheel via a constant gear ratio. Therefore, the operating point of the EM cannot be changed by a gearshift during electric driving or recuperation. In addition, due to its lower power output than the P2 HEV, the P1-EM allows the load point of the ICE to be increased to a lesser extent. This can have a less positive effect on the efficiency of the ICE. Furthermore, operating states may occur in which the load point of the ICE is increased via the P4-EM on the rear axle, which may result in high transmission losses.

With the series-parallel DHT, hybrid operation shows a comparatively good acceleration time of approx. $5.9 \mathrm{~s}$ due to the powerful EM. This means that all variants of this concept reach the traction force limit up to at least $100 \mathrm{~km} / \mathrm{h}$.

However, the variants of the concept show high differences in $\mathrm{CO}_{2}$ emissions. They range between $113 \mathrm{~g} / \mathrm{km}$ and $117.1 \mathrm{~g} / \mathrm{km}$. The transmission ratio between the ICE and the wheel has a major influence, since with increasing the gear ratio, the ICE is operated at higher speeds and lower torques. As a result, a higher amount of load point increase is carried out to improve ICE efficiency. The energy obtained by load point increase must later be used for electric driving or load point decrease to ensure SOC neutrality. The energy available for electrical operation is therefore generated under high losses compared to energy gained from recuperation. Thus, the $\mathrm{CO}_{2}$ emissions are increased. Furthermore, the high power of the EM has a negative effect on $\mathrm{CO}_{2}$ emissions as well. Due to the high power, the EM are operated at a low average power and thus at low efficiencies. As a result, their efficiency during electric driving and recuperation is lower compared to other concepts. This means that fewer driving situations can be covered in electric operation.

Compared to the series-parallel concept, the acceleration times of the PS-DHT variants increase. Depending on the variant, acceleration times between approx. $6.1 \mathrm{~s}$ and $6.3 \mathrm{~s}$ are possible. In contrast to the series-parallel DHT, the driving performance is slightly reduced, because EM2 has a lower maximum power and torque. Therefore, the variants of the PS-DHT do not reach the traction force limit up to speeds of $100 \mathrm{~km} / \mathrm{h}$. 
The $\mathrm{CO}_{2}$ emissions of the PS-DHT are between approx. $115.7 \mathrm{~g} / \mathrm{km}$ and $117.5 \mathrm{~g} / \mathrm{km}$. Therefore, the PS-DHT has a lower efficiency in WTLC compared to the series-parallel DHT. Although the ICE can operate at high efficiencies due to the eCVT mode, the powertrain efficiency during electric driving and during recuperation is lower compared to the series-parallel DHT. This means that less electrical energy is available from recuperation in the traction phase and a higher amount of energy is required for electric driving. This means that the ICE operates more frequently and must provide additional energy by increasing the load point. In addition, powerful EM are necessary for the PS-DHT to meet the driving performance requirements. Thus, they operate at low average loads and have a low average efficiency.

If the PS-DHT is supplemented by a 4-speed transmission, the driving performance and $\mathrm{CO}_{2}$ emissions are reduced. The variants achieve acceleration times between $6.3 \mathrm{~s}$ and $6.6 \mathrm{~s}$ and $\mathrm{CO}_{2}$ emissions between $110.2 \mathrm{~g} / \mathrm{km}$ and $111 \mathrm{~g} / \mathrm{km}$. Due to the lower total electrical power of the EM, the acceleration time increases slightly compared to the PS-DHT. However, four electrical and four power-split operating modes allow adaptation of the operating points of the energy converters to the driving situation. Especially in hybrid operation, this leads to the fact that less power of the ICE must be transmitted via the electrical branch by adjusting the speed of the ring gear. This increases the transmission efficiency and reduces $\mathrm{CO}_{2}$ emissions. Furthermore, the efficiency of the $\mathrm{EM}$ in both traction and thrust phase is positively influenced, which also has a positive effect on energy consumption.

The variants of the MM-DHT with two PGS achieve acceleration times between $6.3 \mathrm{~s}$ and 7.1 $\mathrm{s}$ in hybrid operation. This results in similar driving performance as the PS-DHT with a 4-speed transmission or P2 HEV.

Due to an additional eCVT mode and a parallel mode, the MM-DHT concept with two PGS achieves lower $\mathrm{CO}_{2}$ emissions than the PS-DHT. Although the transmission losses are higher because the concept is mechanically more complex, the additional degrees of freedom regarding the choice of operating points have a positive effect on efficiency. This results in $\mathrm{CO}_{2}$ emissions between $111.2 \mathrm{~g} / \mathrm{km}$ and $114.2 \mathrm{~g} / \mathrm{km}$. Compared to the $\mathrm{P} 2 \mathrm{HEV}$, the $\mathrm{CO}_{2}$ emissions are similarly good.

If the MM-DHT concept is enhanced by an additional PGS, the driving performance and efficiency are reduced compared to the concept with two PGS. The acceleration times are in a range between $6.6 \mathrm{~s}$ and $7.3 \mathrm{~s}$ and the $\mathrm{CO}_{2}$ emissions are between $109.4 \mathrm{~g} / \mathrm{km}$ and $111.8 \mathrm{~g} / \mathrm{km}$. Compared to the concept with two PGS, the number of operating modes increases, so that more efficient operating points of the energy converters can be selected in WLTC. On the one hand, the concept offers four modes in electrical operation in contrast to the concept with two PGS with two modes. On the other hand, more degrees of freedom are also available in hybrid operation with three parallel and four eCVT modes.

The results in Figure 11 show that mechanically complex concepts offer a higher efficiency in the $C$ segment due to additional operating modes. At the same time, they show only slightly increased acceleration times compared to concepts with more powerful EM. Therefore, the concepts P2, MM-DHT with two PGS, PS-DHT with 4-speed transmission and MM-DHT with three PGS offer a better compromise in terms of performance and efficiency in hybrid operation. A higher mechanical complexity leads to a better efficiency.

However, it should be noted that the differences in efficiency and driving performance are small. For example, the best variants of the series-parallel DHT have good $\mathrm{CO}_{2}$ emissions, which are about $1.7 \mathrm{~g} / \mathrm{km}$ to $2 \mathrm{~g} / \mathrm{km}$ higher than the best variants of P2 HEV or MM-DHT with two PGS. In addition, the concepts have to meet high performance requirements in hybrid operation. For example, the concepts must reach the traction force limit between $0 \mathrm{~km} / \mathrm{h}$ and $10 \mathrm{~km} / \mathrm{h}$ even when loaded, while the battery is being charged or the vehicle is driven purely electrically. This leads to a comparatively high total EM power for the PS-DHT and the series-parallel DHT, see Section 6. Due to the high requirements, these two concepts show a lower efficiency and driving performance. It can be assumed that different results are obtained if the driving performance requirements are reduced. 
Furthermore, other important factors such as the required installation space and the costs influence the evaluation of the drive concepts. As these two important variables are not considered in this paper, the concepts are only assessed in terms of efficiency and driving performance.

\subsubsection{Comparison of Efficiency in Hybrid Driving and Driving Performance in Electric Operation}

In addition to the driving performance in hybrid operation, this paper also examines the driving performance in electric operation. Figure 12 shows the $\mathrm{CO}_{2}$ emissions in hybrid operation versus the acceleration time from $0 \mathrm{~km} / \mathrm{h}$ to $100 \mathrm{~km} / \mathrm{h}$ in electric operation.

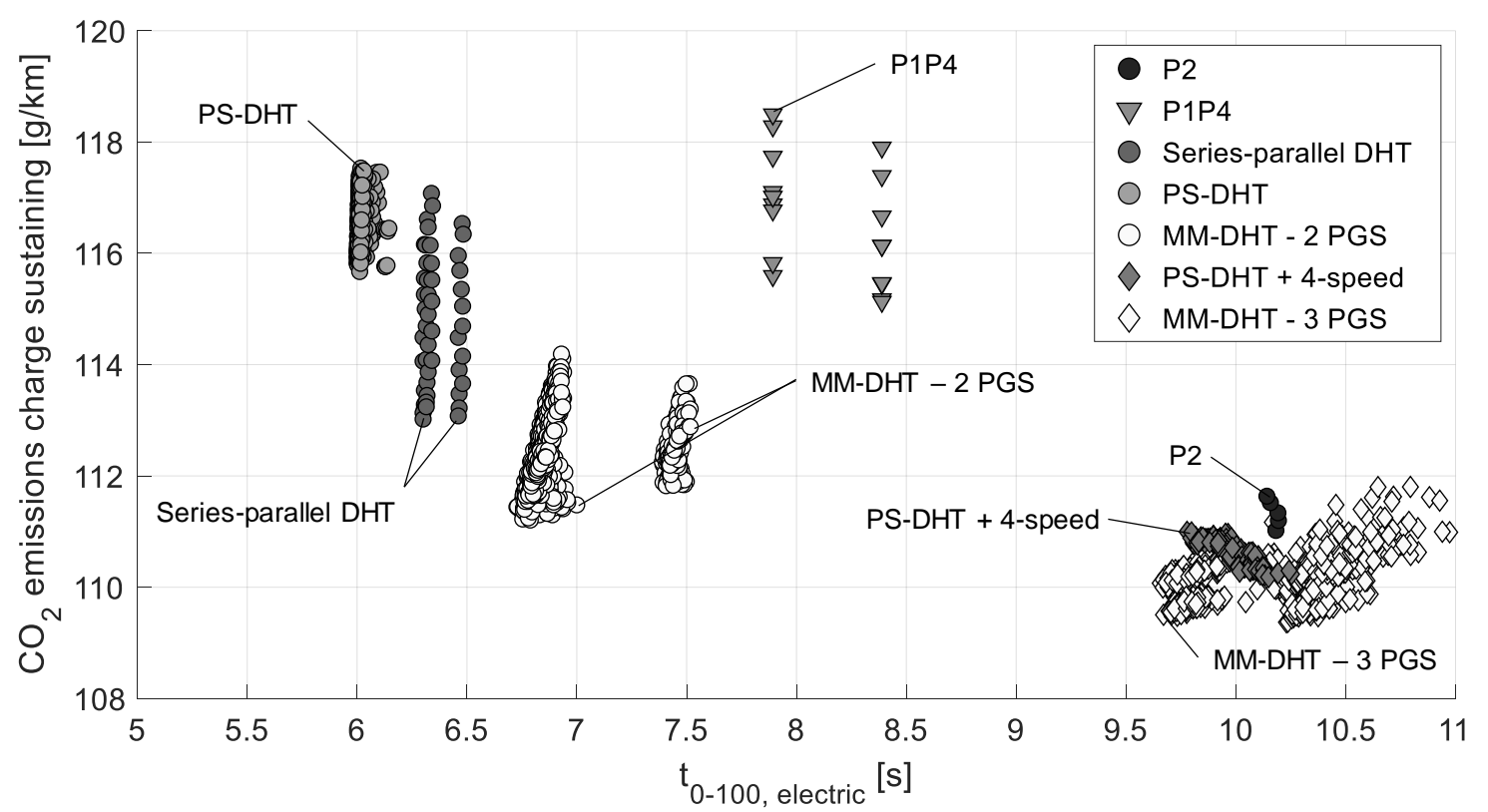

Figure 12. Comparison of charge sustaining efficiency in WLTC and electric driving performance for drive train concepts compliant with $\mathrm{C}$ segment requirements.

It can be seen that concepts with a high total electrical power achieve lower acceleration times in electrical operation. The variants of the PS-DHT and series-parallel DHT have acceleration times of approx. $6 \mathrm{~s}$ to $6.5 \mathrm{~s}$, as the EM have total outputs of $170 \mathrm{~kW}-190 \mathrm{~kW}$ and $270 \mathrm{~kW}-290 \mathrm{~kW}$, respectively. It should be noted that the variants of the PS-DHT have lower acceleration times, although less EM power is installed. The reason for this is that with the PS-DHT, both EM can drive the vehicle in electric mode because of the OWC between the ICE and PGS. In contrast, in series-parallel DHT only the EM2 can drive the vehicle.

The lower the power available in electrical operation, the higher the acceleration time. Therefore, the acceleration time of the MM-DHT with two PGS is between $6.6 \mathrm{~s}$ and $7.5 \mathrm{~s}$. In addition, the results show that two different total EM powers are considered. In case of the P1P4 concept, the acceleration time increases to $7.9 \mathrm{~s}$ or $8.4 \mathrm{~s}$. For mechanically more complex concepts, EM powers of $80 \mathrm{~kW}$ to 90 $\mathrm{kW}$ are required. For the P2 hybrid, this results in an acceleration time of about $10.1 \mathrm{~s}$. For the PS-DHT with 4-speed transmission, the acceleration time increases to $9.8 \mathrm{~s}$ to $10.3 \mathrm{~s}$. The largest differences are found in the MM-DHT with three PGS. The variants of this concept achieve acceleration times between $9.7 \mathrm{~s}$ and $11 \mathrm{~s}$.

In comparison to hybrid operation with a charged battery, these acceleration times therefore differ by approx. $3.1 \mathrm{~s}$ in case of the MM-DHT with three PGS and $3.8 \mathrm{~s}$ for the P2 HEV. For the P1P4 the difference is also $3.8 \mathrm{~s}$. Furthermore, the difference is considerably smaller for the mechanically simple concepts with high total electrical power. The MM-DHT with two PGS offers a good compromise between the acceleration time in hybrid operation and in electrical operation. For this concept, the acceleration time in electrical operation is $0.5 \mathrm{~s}$ higher. 
The results in Figure 12 therefore show that the mechanically complex concepts have lower driving performance in electrical operation than the concepts with a simpler mechanical design because of their lower total electrical power. However, it should be noted that no requirement regarding the acceleration time was specified for electrical operation. In this case, it can be assumed that the mechanically more complex concepts would achieve this acceleration time with a lower total electrical power.

\subsubsection{Comparison of Hybrid Driving Performance with Charged and Discharged Battery}

In addition to electrical operation, the consistency of the acceleration behavior also plays a role in the evaluation of the concepts. Therefore, the concepts in this section are examined regarding the difference between charged and discharged battery in hybrid operation. For this purpose, Figure 13 shows the corresponding acceleration times from $0 \mathrm{~km} / \mathrm{h}$ to $100 \mathrm{~km} / \mathrm{h}$. A solid black line shows the range of equal driving performance with fully charged and discharged battery in hybrid mode. To the right of this line, the driving performance is better with a discharged battery than with a charged battery. Furthermore, isolines indicate a constant time difference between the operation with charged and discharged battery.

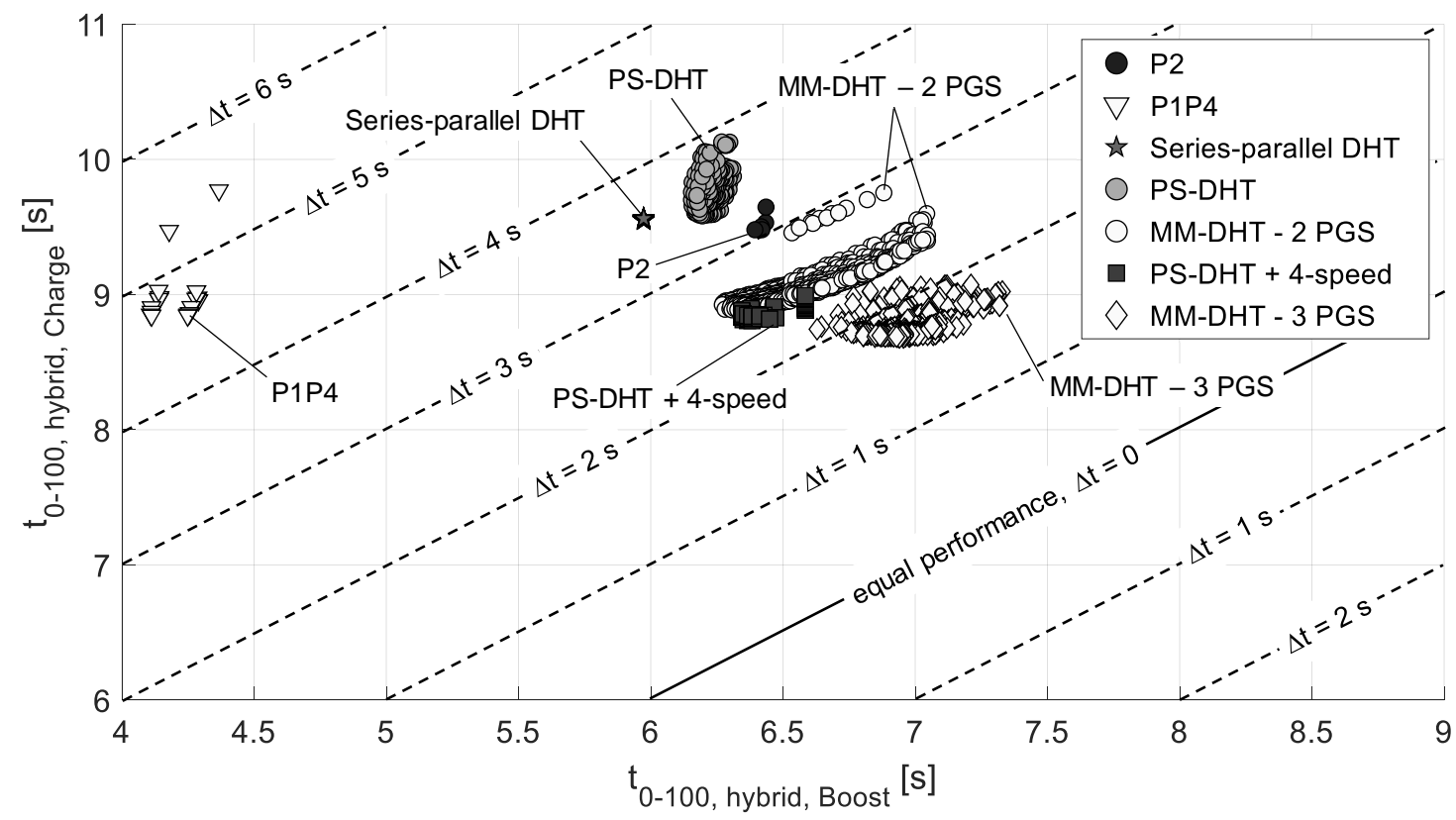

Figure 13. Comparison of acceleration time from $0 \mathrm{~km} / \mathrm{h}$ to $100 \mathrm{~km} / \mathrm{h}$ in hybrid operation in case of charging or discharging the battery.

The highest difference between hybrid operation with charged and discharged battery occurs in case of the P1P4 HEV. At high SOC, additional drive power is available at the rear axle of the vehicle resulting in a good traction potential. However, in case of a low SOC, drive power is only available at the front axle. Thus, the difference in acceleration time is more than $4 \mathrm{~s}$. Therefore, the concept has disadvantages regarding the similarity of driving performance. Furthermore, Figure 13 shows that some variants of the P1P4 HEV have a shorter acceleration time at low SOC than the variants of the P2 HEV. This is due to the possibility to transfer a part of the drive power in series mode to the rear axle of the vehicle. The P1-EM works as a generator, while the P4-EM can provide its power at the rear axle with losses. In the low speed range, when the traction force limit of the front axle is reached, the P1P4 concept can thus offer advantages over the P2 HEV.

A smaller difference results in case of the series-parallel DHT and the PS-DHT. Their acceleration times differ by more than $3 \mathrm{~s}$ and less than $4 \mathrm{~s}$. The P2 hybrid can be rated slightly better. A disadvantage of the series-parallel DHT is that the drive power of the ICE must be transmitted in series mode in a certain speed range, depending on the gear ratio between ICE and wheel. This can result in high 
conversion losses, so that the transmission efficiency declines and less drive power is available at the wheel.

A slightly better compromise than P2 HEV is offered by the MM-DHT variants with two PGS. With this concept the difference in acceleration time of the hybrid operation at high and low SOC is less than $3 \mathrm{~s}$. Compared to the PS-DHT, the two eCVT modes as well as the parallel mode have a positive effect on the acceleration time, since less drive power has to be transmitted via the EM. This means that a higher proportion of the drive power is transmitted to the wheel mechanically, resulting in lower power losses.

If additional mechanical components are added to the PS-DHT, the difference between high and low SOC is reduced. The 4-speed transmission enables transmission of a higher share of the drive power mechanically in eCVT mode, resulting in shorter acceleration times compared to the PS-DHT. For the MM-DHT with two PGS there are similar acceleration times and differences between the two states of charge.

The lowest difference in acceleration time is shown by the MM-DHT variants with three PGS. Depending on the dimensioning, the difference is slightly higher than $2 \mathrm{~s}$ and in the best case only about $1.5 \mathrm{~s}$. Since this concept offers the highest number of operating modes, the difference in driving performance is the lowest.

The results in Figure 13 therefore lead to the conclusion that the increase in mechanical effort in the transmission leads to less difference in driving performance in hybrid operation at high and low SOC. The additional operating modes provided by additional mechanical components can lead to a higher share of the drive power provided by the ICE being transferred mechanically to the wheel, resulting in better driving performance at low SOC. As a result, the difference in hybrid operation is lower and the driving performance is better. It should be noted that the MM-DHT with three PGS and the PS-DHT with 4-speed transmission offer better performance in comparison to the P2 HEV in case of a low SOC. This is due to the four eCVT modes offered by both concepts. Compared to the P2 HEV with 8-speed DCT, they provide better adaptation to the traction force hyperbola, resulting in lower acceleration times. In addition, there are variants of the MM-DHT with two PGS, which offer advantages compared to the P2 HEV.

An increase in performance in hybrid operation at low SOC would only be possible in concepts with low mechanical effort if the EM could achieve better efficiencies or if the ICE offered a higher maximum power.

It should be noted that no explicit requirements were made for a small difference in acceleration times. In this case, the required total electrical power of the concepts would differ from the results shown in this paper.

\subsection{Evaluation of Conversion Quality and Transmission Efficiency}

For further evaluation of the concepts in hybrid operation, the average transmission efficiency and the average conversion quality in WLTC are calculated. The conversion quality is introduced in [11] and describes the ratio of the theoretical optimum efficiency of the ICE to the actual efficiency of the ICE. The parameter thus describes the ability of a transmission to operate an energy converter at its power-specific optimum efficiency.

Figure 14 shows the average conversion quality and the average transmission efficiency in hybrid operation in the WLTC. There are differences between add-on hybrid concepts and DHT on the one hand and between mechanically simple and complex concepts on the other hand. 


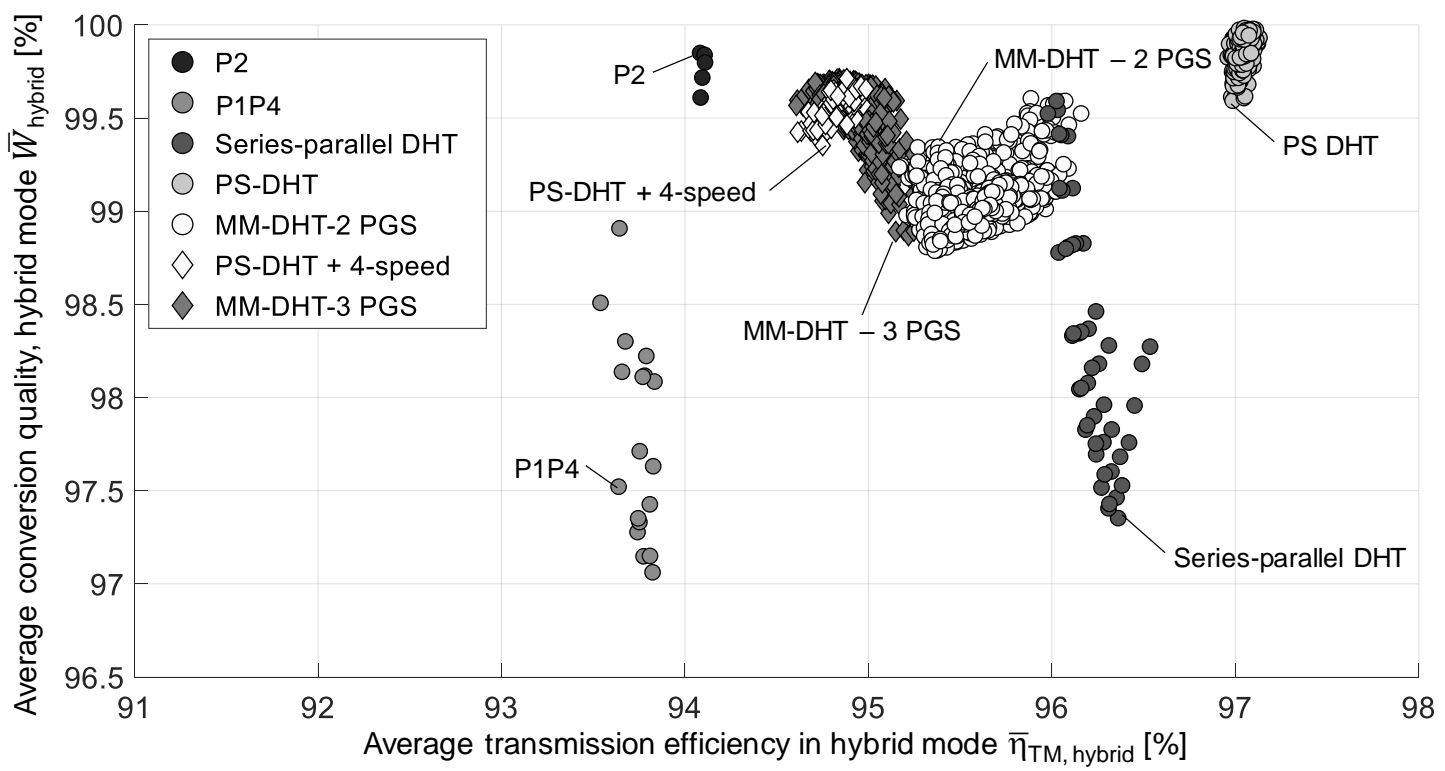

Figure 14. Comparison of average conversion quality of the ICE and average transmission efficiency in hybrid operation in WLTC.

The variants of the PS-DHT achieve very good transmission efficiencies of around $97 \%$ in hybrid operation. A positive effect is that the transmission is mechanically simple, so that mechanical losses are low. In addition to a good transmission efficiency, all variants of the PS-DHT achieve high conversion qualities of over $99.5 \%$. This is due to the eCVT operating mode, which allows infinite variation of the transmission ratio, so that the ICE can operate at its power-specific optimum efficiency operating point.

Compared to the PS-DHT, the variants of the series-parallel DHT have lower transmission efficiencies in hybrid operation. Although this concept also has a simple mechanical design and thus low mechanical losses, the EM are much more powerful. Since the conversion losses in the EM are taken into account in the transmission efficiency in case of serial operation, the efficiency is lower compared to the variants of the PS-DHT. In addition, the transmission efficiency in hybrid operation depends on the wheel power in which the hybrid operation occurs. It is thus possible that hybrid operation in case of PS-DHT takes place at higher wheel power, whereby the transmission efficiency tends to be better, since load-independent losses cause a smaller share of the losses.

In contrast to the PS-DHT, the variants of the series-parallel DHT have lower conversion qualities in hybrid operation. Compared to the other concepts, there are greater differences. In the best case, the conversion quality is over $99.5 \%$ and in the worst case less than $97.5 \%$. This variation results from the scaling of the FD ratios. An increase in the FD ratio leads to a lower conversion quality, since the ICE is operated at higher speeds and lower torques due to the constant gear ratio to the wheel. Thus, load point increase of the ICE is necessary to increase the efficiency of the ICE. Since the load point increase does not lead to an increased efficiency in all areas, the average conversion quality decreases. Furthermore, an increase in the FD ratio means that lower speeds can be achieved in parallel mode. Therefore, there may be variants in which the serial mode must be selected at high speeds. Since in serial mode both the speed and the torque of the ICE can be chosen freely, the conversion quality is positively influenced in serial mode.

Compared to the mechanically simple DHT, the P2 HEV and P1P4 HEV add-on hybrids achieve lower average transmission efficiency. In case of the P2 HEV it is over $94 \%$ and in case of the P1P4 it is around $93.5 \%$. The reason for this is that mechanical losses are higher due to the more complex design.

Regarding the average conversion quality, there are greater differences. The P2 HEV variants achieve similarly good conversion qualities as PS-DHT of over $99.5 \%$. On the one hand, the combination of gear ratios and ICE efficiency map has a positive effect on the conversion quality. On the other hand, 
with the P2 HEV it is possible to operate the ICE in more favorable efficiency ranges by increasing the load point.

In contrast, the extent of load point increase is limited for the P1P4 HEV. On the one hand, the P1-EM only has a power of $15 \mathrm{~kW}$. On the other hand, the load point increase via the P4-EM is associated with high losses. For this reason, the FD ratio of the P1P4 HEV variants also has a strong influence on the average conversion quality. As with the series-parallel DHT, an increase of the FD ratio leads to a reduction of the conversion quality of the ICE.

A good compromise between conversion quality and transmission efficiency in hybrid operation is offered by the mechanically complex DHT. The variants of the MM-DHT with two PGS achieve transmission efficiencies between approx. $95 \%$ and $96.1 \%$ in hybrid operation with conversion qualities of $98.7 \%$ to $99.6 \%$. With a higher mechanical complexity as with the MM-DHT with three PGS, the transmission efficiency declines to a range between $94.6 \%$ and $95.3 \%$. However, the number of operating modes gained through the higher mechanical complexity leads to an increase in the conversion quality compared to the MM-DHT with two PGS. It lies between approx. $98.7 \%$ and $99.7 \%$.

The extension of the PS-DHT by a 4-speed transmission has a negative effect on the average transmission efficiency in hybrid operation. It lies between $94.6 \%$ and $95 \%$ and is significantly lower than in case of the PS-DHT. The conversion quality is also reduced compared to the PS-DHT. However, it is still high with about $99.3 \%$ to $99.7 \%$. It should be noted that the local optimal operation strategy approach always achieves an optimal compromise between high conversion quality of the ICE and low losses in the EM. Therefore, a lower conversion quality of the ICE does not directly lead to higher $\mathrm{CO}_{2}$ emissions, see Figure 11.

The results in Figure 14 show characteristic differences between different types of hybrid transmission concepts. For example, mechanically simple DHT such as PS-DHT or series-parallel DHT in hybrid operation show very high transmission efficiencies. The concepts have a low mechanical complexity, so that mechanical losses are reduced. In addition to a high transmission efficiency, these concepts can achieve high conversion qualities of over $99 \%$. However, as the minimum required total EM power in Figure 10 shows, powerful EM are necessary. DHT with increased mechanical complexity have lower transmission efficiencies in hybrid operation, but these concepts can achieve comparably high conversion qualities as mechanically simpler concepts, so that with reduced total EM power, lower $\mathrm{CO}_{2}$ emissions are achieved in SOC-neutral operation. Therefore, especially MM-DHT are a good solution. Although add-on-HEV concepts can also achieve high conversion qualities, their transmission efficiency is lower due to the higher mechanical effort.

\section{Summary}

The electrification of the drive train creates considerable degrees of freedom in the design of drive trains. Depending on the number and positioning of EM, there may be significant differences regarding the topology and functionality of the concepts. On the one hand, additional EM can supplement conventional drive trains. Common solutions to add-on concepts are P2 HEV as well as electrified all-wheel drive systems such as P1P4 HEV. In addition, it is also possible to implement DHT. These transmissions are dedicated to HEV and allow different operating modes than parallel hybrids. Depending on the structure, DHT can be of varying complexity.

In this paper, various powertrain concepts, which differ in their design and operating modes, are examined. These include parallel hybrids, series-parallel DHT, PS-DHT, and MM-DHT with different mechanical complexity. In this paper, the concepts are briefly described regarding their structure and operating modes. Furthermore, the mechanical complexity of the concepts is estimated and quantified by considering mechanically relevant components.

To compare powertrain concepts driving performance requirements in different speed ranges are defined for a C, D, and E segment vehicle. These include reaching the traction force limit in the low speed range, requirements in the medium speed range, which are quantified by boost or charge power, and maximum speed requirements. 
A comparatively small number of requirements is defined to allow for short computing times while at the same time allowing for a high number of powertrain variants. Therefore, a full factorial scaling plan can be used so that the system behavior can be identified.

The consideration of different speed ranges also ensures that the delivery maps of the concepts match realistic demand maps of the drive concepts.

The requirements are defined for different drive train states to take into account the interaction of ICE and battery. In hybrid operation, a distinction is made as to whether the battery can be discharged or needs to be charged. In addition, requirements for electrical operation are defined.

Using a modular simulation environment described in this paper, variants that meet the requirements can be identified based on a driving performance simulation. Differences arise especially regarding the required EM power. This differs between the segments, since the tractive forces resulting from the requirements depend on vehicle and drive train parameters. Furthermore, concept-specific differences regarding the required EM power are analyzed. This electrical effort is compared to the quantified mechanical effort.

The results show that a reduction in the number of mechanical components requires comparatively high EM power. Responsible for this is a constant gear ratio between the EM, which in most situations operates as a motor. This EM must, on the one hand, reach the maximum speed of the vehicle in hybrid operation and, on the other hand, enable reaching the traction force limit both in hybrid operation and electric driving. Therefore, this EM must have a comparatively high torque and thus a high power output. In addition, due to their structure, the concepts with low mechanical effort must transmit a high share of the drive power electrically. In case of the series-parallel DHT, the serial mode must be selected in a certain speed range when the battery is discharged. In this case, the drive power is only provided by the ICE and transmitted via both EM. Depending on the gear ratio, the PS-DHT also needs to transmit a certain share of the drive power via the EM. The fact that an EM must receive the power of the ICE and therefore operate as a generator results in a dependency on the maximum power of the ICE.

For these reasons, the required EM power of the mechanical simple concepts increases distinctly with higher vehicle segments or higher requirements. In these cases, an increase in the mechanical complexity of the transmission is advisable. Additional components allow transmission of higher shares of the drive power mechanically, which can reduce the required EM power. In addition, several gears may be available for the EM, which is usually operating as a motor. Mechanically complex concepts are therefore better suited for use in higher vehicle segments. Depending on the segment and the EM used, they do not require significantly more EM power than concepts with one EM, such as P2 HEV.

The comparison of the required EM power shows that in the $C$ segment there are the least differences between the drive concepts. For this reason, hybrid operation is examined within a benchmark analysis. This paper focuses on efficiency and performance since both costs and the required installation space can only be evaluated with comparably high uncertainty in early concept phases. To assess the efficiency, the SOC-neutral $\mathrm{CO}_{2}$ emissions in the WTLC are considered. To compare the driving performance, the acceleration time from $0 \mathrm{~km} / \mathrm{h}$ to $100 \mathrm{~km} / \mathrm{h}$ for hybrid operation and electric driving is analyzed. The results show that mechanically complex concepts offer a good compromise between driving performance and efficiency in hybrid operation. On the one hand, they achieve low $\mathrm{CO}_{2}$ emissions. On the other hand, they show small differences between the acceleration times of hybrid operation with charged and discharged battery.

In contrast, the $\mathrm{CO}_{2}$ emissions of the mechanically simpler concepts increase. Regarding hybrid operation, they therefore do not represent more attractive solutions in the context of these investigations. However, it should be noted that due to the higher electrical power, the driving performance in electric operation increases. In addition, there are only minor differences in the acceleration time during hybrid operation with a charged battery and electric operation. 
To be able to derive concept-specific properties, the average transmission efficiency and the conversion quality in the WLTC are also investigated in hybrid operation. The results show that mechanically simple concepts such as PS-DHT or series-parallel DHT can provide both good transmission efficiency and high conversion quality. However, the PS-DHT offers advantages due to the eCVT mode and can achieve higher conversion qualities.

With mechanically more complex DHT such as MM-DHT with two or three PGS or a PS-DHT with a 4-speed transmission, the average transmission efficiency and conversion quality decline slightly. Compared to the mechanically simpler concepts, however, considerably less electrical power must be installed in the drive train. Therefore, these concepts offer the best compromise between transmission efficiency and conversion quality.

The results also show that the considered parallel hybrids have the lowest average transmission efficiencies in hybrid operation. In case of the P2 HEV, however, a very high conversion quality can be achieved with lower required EM power.

Author Contributions: C.S. and F.K.; methodology, C.S.; software, C.S.; validation, C.S., F.K.; formal analysis, C.S.; investigation, C.S. and F.K.; resources, C.S.; data curation, C.S.; writing-original draft preparation, C.S.; writing-review and editing, C.S.; visualization, C.S.; supervision, F.K. All authors have read and agreed to the published version of the manuscript.

Funding: This research received no external funding

Conflicts of Interest: The authors declare no conflict of interest.

\section{References}

1. Lange, A. Optimierung modularer Elektro- und Hybridantriebe. Ph.D. Dissertation, Technische Universität Braunschweig, Braunschweig, Germany, 6 February 2018.

2. Suzuki, Y.; Nishimine, A. Development of New Plug-In Hybrid Transaxle for Compact-Class Vehicles; SAE International: Warrendale, PA, USA, 2017.

3. Imamura, T.; Tabata, A. Concept and Approach of Multi Stage Hybrid Transmission; SAE International: Warrendale, PA, USA, 2017.

4. Kato, S.; Ando, I. Development of Multi Stage Hybrid System for New Lexus Coupe. SAE Int. J. Alt. Power 2017, 6, 136-144. [CrossRef]

5. Conlon, B.; Blohm, T. The Next Generation "Voltec" Extended Range EV Propulsion System; SAE International: Warrendale, PA, USA, 2015.

6. Holmes, A.; Liu, J. General Motors Electric Variable Transmission for Cadillac CT6 Sedan; SAE International: Warrendale, PA, USA, 2016.

7. Lange, A.; Küçükay, F. A new, systematic approach to determine the global energy optimum of a hybrid vehicle. Automot. Engine Tech. 2016, 1, 35-46. [CrossRef]

8. Lange, A.; Küçükay, F. Ermittlung des globalen energetischen Optimums von Hybridantrieben. ATZ 2017, 119, 106-111. [CrossRef]

9. Sciaretta, A.; Guzzella, L. Control of Hybrid Electric Vehicles. IEEE Control Syst. Mag. 2007, 27, 60-70.

10. Sciaretta, A.; Back, M.; Guzzella, L. Optimal Control of Parallel Hybrid Electric Vehicles. IEEE Trans. Control Syst. Technol. 2004, 12, 352-363. [CrossRef]

11. Lange, A.; Küçükay, F. Benchmark parameters of transmissions. Automot. Engine Tech. 2018, 3, 1-10. [CrossRef]

(C) 2020 by the authors. Licensee MDPI, Basel, Switzerland. This article is an open access article distributed under the terms and conditions of the Creative Commons Attribution (CC BY) license (http://creativecommons.org/licenses/by/4.0/). 